\title{
The strategic control of prospective memory monitoring in response to complex and probabilistic contextual cues
}

\author{
Julie M. Bugg ${ }^{1} \cdot$ B. Hunter Ball ${ }^{1}$
}

Published online: 8 March 2017

(C) Psychonomic Society, Inc. 2017

\begin{abstract}
Participants use simple contextual cues to reduce deployment of costly monitoring processes in contexts in which prospective memory (PM) targets are not expected. This study investigated whether this strategic monitoring pattern is observed in response to complex and probabilistic contextual cues. Participants performed a lexical decision task in which words or nonwords were presented in upper or lower locations on screen. The specific condition was informed that PM targets ("tor" syllable) would occur only in words in the upper location, whereas the nonspecific condition was informed that targets could occur in any location or word type. Context was blocked such that word type and location changed every 8 trials. In Experiment 1, the specific condition used the complex contextual cue to reduce monitoring in unexpected contexts relative to the nonspecific condition. This pattern largely was not evidenced when the complex contextual cue was probabilistic (Experiment 2). Experiment 3 confirmed that strategic monitoring is observed for a complex cue that is deterministic, but not one that is probabilistic. Additionally, Experiments 1 and 3 demonstrated a disadvantage associated with strategic monitoring - namely, that the specific condition was less likely to respond to a PM target in an unexpected context. Experiment 3 provided evidence that this disadvantage is attributable to impaired noticing of the target. The novel findings suggest use of a complex contextual cue per se is not a boundary condition for the strategic, context-specific allocation of monitoring processes to support prospective remembering; however, strategic monitoring is
\end{abstract}

Julie M. Bugg

jbugg@wustl.edu

1 Washington University in St. Louis, CB 1125. 1 Brookings Drive, St. Louis, MO 63130, USA constrained by the predictive utility of the complex contextual cue.

Keyword Prospective memory · Strategic monitoring · Attention · Context specific · Probabilistic

Anyone who has tried to remember to give a message to a professor or, conversely, a student, has likely experienced what prospective memory (PM) researchers refer to as "monitoring" in service of an event-based PM intention. Monitoring involves holding in mind an intention and actively checking the environment for a cue (i.e., target) that signals it is the appropriate time to fulfill the intention (Guynn, 2003; Smith, 2003). There is agreement among theorists that monitoring is a resource-demanding process that incurs costs to performance of ongoing activities (e.g., McDaniel \& Einstein, 2000; Scullin, McDaniel, \& Shelton, 2013; Smith, 2003; Smith \& Bayen, 2004). Accordingly, an important question concerns the ability to monitor strategically - that is, to heighten monitoring when contextually appropriate and to relax monitoring when it is not needed, thereby conserving limited resources (cf. Einstein, McDaniel, Williford, Pagan, $\&$ Dismukes, 2003). Although research on this topic is relatively scarce, several studies have provided initial evidence for strategic monitoring in response to simple contextual cues (see Smith 2016 for a review). The purpose of this study was to extend this work by examining three questions of theoretical and practical importance: Do individuals show evidence of strategic monitoring in response to complex contextual cues? Is strategic monitoring observed when cues are probabilistic, as may often exist in real-world situations? These first two questions address potential boundary conditions for strategic monitoring, that is, conditions under which strategic monitoring might not be observed. Finally, although we embrace the 
perspective that strategic monitoring typically represents optimal behavior, a third question that was addressed is whether strategic monitoring may come at a potential disadvantage to PM performance.

\section{Prior evidence of strategic monitoring in PM tasks}

In PM studies, monitoring is routinely observed for nonfocal cues, which refer to PM targets that may not otherwise be detected as part and parcel of engaging processes required for the ongoing task. For example, a syllable (e.g., "tor") that serves as a PM target in an ongoing lexical decision task is considered a nonfocal cue (whereas a word such as tornado would be considered focal; see Einstein \& McDaniel, 2005). Monitoring is inferred by comparing response times (RT) on non-PM target trials during a baseline block in which participants perform only the ongoing task (e.g., lexical decision) to a PM block in which they perform the ongoing task in the presence of a PM intention. The difference is referred to as "cost," and it reflects the interference to ongoing task performance (i.e., slowing of RTs) when attention is allocated to the PM task (Smith, 2003).

Strategic monitoring may be defined as deployment of attention in the service of detecting a PM target according to a "policy" that is established in advance of (i.e., based on instructions) or during task performance, and which exploits information about the likelihood that a PM target will occur within a given context. According to Guynn's (2003) twoprocess model of strategic monitoring, contextual cues may be used to initiate a prospective retrieval mode in which the intention is activated and there is a readiness to perform the PM response. Such a mode is thought to operate across all trials of an ongoing task, and its maintenance induces cost. Contextual cues may also be used to modulate the targetchecking process, which can be a more intermittent and selective process that is used to verify whether a given stimulus includes intention-relevant information (e.g., is the PM target). Target checking also induces cost. Variation in cost across contexts in which PM targets are expected (i.e., high likelihood of occurrence) versus unexpected (i.e., low likelihood of occurrence) is indicative of strategic monitoring.

In an initial study that demonstrated strategic monitoring of an event-based PM intention, a three-phase PM task was administered. Participants were told to expect that PM targets would occur selectively within Phase 3 (Marsh, Hicks, \& Cook, 2006; see also Ball, Brewer, Loft \& Bowden, 2014; Knight et al., 2011). Phases 1 and 3 comprised an ongoing lexical decision task, thereby allowing for a comparison of cost between the phase in which PM targets were not expected and the phase in which they were expected. As predicted, relative to a control group with no intention, cost for participants in the PM group was observed selectively in Phase 3.
This finding provided evidence that monitoring resources may be deployed in a context-specific fashion (cf. Meier, Zimmermann, \& Perrig, 2006, for a similar finding of an increase in self-reported monitoring in a task in which PM targets were expected). Applying Guynn's (2003) model, one interpretation is that context was used to initiate (Phase 3) or not initiate (Phase 1) a prospective retrieval mode. This raises the question of whether strategic monitoring is evidenced when context changes occur more rapidly (e.g., every $x$ trials) or unpredictably (i.e., randomly trial to trial). In such circumstances, participants may remain in a retrieval mode across trials (contexts) such that differences in cost between expected and unexpected contexts provide evidence that participants strategically modulate the extent of target checking based on contextual cues.

Lourenço, White, and Maylor (2013) recently tackled this question by examining strategic monitoring in conditions in which the expected and unexpected contexts were randomly intermixed on a trial-by-trial basis (i.e., random $P M$ procedure). In an initial baseline block, all participants performed an ongoing lexical decision task. In a subsequent block, a control condition continued to perform only the ongoing task, whereas participants in two PM conditions were told to press a special key whenever they encountered the syllable "tor" (the PM target) during the lexical decision task. Most critically, participants in the specific condition were instructed that PM targets would occur in word stimuli (i.e., the expected context), but not nonword stimuli (i.e., the unexpected context). In contrast, those in the nonspecific condition were instructed that PM targets could occur in either words or nonwords. Words and nonwords were randomly intermixed during both blocks. The key finding was that cost was equivalent for the specific and nonspecific conditions for word trials, but cost was significantly reduced for the specific compared to the nonspecific condition on nonword trials. This strategic monitoring pattern indicates that participants flexibly heightened and relaxed monitoring in response to contextual changes that occurred relatively rapidly and unpredictably (see also Cohen, Jaudas, Hirschhorn, Sobin, \& Gollwitzer, 2012; Kuhlmann \& Rummel, 2014; Lourenço \& Maylor, 2014). Providing insight into the nature of the monitoring process, a second key finding was that cost was not fully eliminated for nonword trials in the specific condition. Because target checking was of no utility in the unexpected context (on nonword trials), this finding suggests that participants in the specific condition remained in a prospective retrieval mode across all trials - those appearing in the expected and the unexpected context. The implication is that the strategic monitoring pattern was likely reflective of variation in a more local target-checking process.

Inspired by the views of monitoring developed by Smith (2003) and Guynn (2003), Lourenço and colleagues (2013, 2014) posited that the PM demands set forth by the instructions in the specific condition led participants to allocate more 
attention to target checking on word trials than on nonword trials, thereby freeing up resources for engaging in the ongoing task on the latter trials. The importance of attentional resources in guiding strategic monitoring was further highlighted by the contrasting pattern of results that was found when the random PM procedure (i.e., contexts randomly intermixed trial to trial) was contrasted with a blocked PM procedure (Lourenço \& Maylor, 2014). In the blocked PM procedure, participants encountered eight trials in the expected context followed by eight trials in the unexpected context, and so on. The key finding was that the strategic monitoring pattern was significantly more pronounced in the blocked PM procedure. According to Lourenço and Maylor (2014), blocking the context lowered demands on attentional control relative to the random PM procedure that required participants to identify the context and rapidly adjust monitoring in an unpredictable fashion. This study aimed to further examine how attentional factors influence strategic monitoring, first by examining strategic monitoring in response to a complex contextual cue presumed to place greater demands on attention than the simple cues used in past studies, and then by examining whether individuals engage costly attentional resources to monitor strategically in the face of less predictable contextual cues.

\section{Experiment 1}

The primary purpose of Experiment 1 was to examine whether strategic monitoring in a blocked PM condition is also evidenced in response to complex contextual cues. A complex contextual cue refers to a cue that represents the co-occurrence of two contextual features, such as a particular identity and location (cf. Smith, Hunt, \& Murray, 2016, for use of simple location cues). There were two primary reasons for using complex contextual cues in this study. First, complex contextual cues afford an opportunity to further examine how the attentional demands associated with detection and identification of the contextual cue affect evidence for strategic monitoring. In particular, participants hold in mind and attempt to identify contexts as expected or unexpected based on the cooccurrence of context features, a process that is presumed to be more attentionally demanding than searching for a single feature, as in the case of a simple contextual cue (see, e.g., Treisman \& Gelade, 1980, for evidence that detection of feature conjunctions is more attentionally demanding than detection of feature singletons). Second, complex contextual cues are likely common in real-world contexts such that their examination may speak to the ubiquity of strategic monitoring outside of the lab, yet no prior strategic monitoring study has examined a complex cue. For instance, consider the earlier example of remembering to give a professor a message, now assuming that the professor is female. An effective strategy would be to initiate a retrieval mode in locations in which the professor is expected to appear (e.g., near or in classroom in which she teaches or building that houses her office) and target check selectively when encountering females. Conversely, these monitoring processes could be relaxed for all individuals appearing in locations where the professor is unexpected (e.g., student recreation center or a dorm), including females.

To provide an initial test of the utility of complex contextual cues for guiding strategic monitoring in Experiment 1, we used a variant of the blocked PM procedure (Lourenço \& Maylor, 2014) wherein the context changed every eight trials. Because there were two contextual dimensions, location (upper vs. lower) and word type (word vs. nonword), there were four possible contexts (i.e., upper word, upper nonword, lower word, and lower nonword). Therefore, the occurrence of any given context was less predictable than in the blocked PM condition in Lourenço and Maylor (2014). Critically, participants in the specific condition were informed that the PM target (the syllable "tor") would occur only in word stimuli that appeared in the upper location on screen during an ongoing lexical decision task. Hereafter, we use the term expected context to refer to blocks of trials in which stimuli were words in the upper location. In contrast, the nonspecific condition was informed that the PM target could occur in either words or nonwords and in either the upper or lower location.

On the one hand, it may be predicted that strategic monitoring would not be evidenced when the blocked PM procedure is combined with a complex contextual cue, thus revealing a potential boundary condition for strategic monitoring. This prediction is based on the assumption that searching for and detecting the complex contextual cue should be attentionally demanding (in comparison to simple contextual cues; cf. Prinzmetal, Presti, \& Posner, 1986; Treisman \& Gelade, 1980), and the demands may interfere with participants' ability to modulate monitoring in a context-specific fashion, akin to how the more attentionally demanding random PM procedure interfered with strategic monitoring for simple contextual cues (Lourenço \& Maylor, 2014). On the other hand, the additional demands associated with the complex contextual cue might be mitigated by use of the blocked PM procedure, which provides environmental support (i.e., a broader window of opportunity) to facilitate participants' identification of the context and adjustment of attention on a block-by-block basis. As such, strategic monitoring may be evidenced just as in the prior study that employed a blocked PM procedure with a simple contextual cue (Lourenço \& Maylor, 2014). That is, there would be equivalent cost for the specific and nonspecific conditions in the expected context (i.e., upper word blocks), but less cost for the specific than for the nonspecific condition in all other contexts (upper nonword, lower word, and lower nonword blocks). (Hereafter we refer to the latter three contexts as unexpected contexts, with this label reflecting exclusively the perspective of the 
specific condition. The nonspecific condition expected PM targets to occur in these contexts as well as the formally labeled "expected" context.)

A second purpose was to examine whether strategic monitoring (if observed), and in particular the specific condition's relaxation of monitoring in unexpected contexts, might come at a potential disadvantage to PM performance. Lourenço and Maylor (2014) examined a similar question by presenting trials on which the PM target appeared in an unexpected context and found that although these targets were missed, they did produce slowing (i.e., suggesting they may have been noticed; Knight et al., 2011). Although embedding such trials intermittently throughout the task offered the advantage of providing multiple observations per condition, a disadvantage of this approach was that it disrupted strategic monitoring (i.e., it was weaker in conditions that included such targets; Lourenço \& Maylor, 2014).

Therefore, we adopted an alternative approach to assessing a potential disadvantage associated with strategic monitoring. A single PM target (the syllable "tor") was presented in a final block of lower nonword trials, the unexpected context that shared no overlapping features with the expected context, and PM target detection was measured. We reasoned that if participants in the specific condition successfully relax monitoring in the unexpected contexts (including the lower nonword context), they should be unlikely to detect a PM target that occurs in a nonword in the lower location (cf. data from a time-based PM task; Cook, Marsh, \& Hicks, 2005). In contrast, those in the nonspecific condition should presumably be monitoring in all contexts and therefore be more likely to detect a PM target that occurs in a nonword in the lower location.

\section{Method}

Design and participants The design was a $3 \times 2 \times 2 \times 2$ mixed factorial, with condition (specific, nonspecific, control) as a between-subjects factor and block (baseline, PM), word type (word, nonword), and location (upper, lower) as withinsubjects factors. Participants ages 18 to 25 years were undergraduates at Washington University in St. Louis, taken from the psychology subject pool. One hundred and eleven participants were randomly assigned to the specific $(n=37)$, nonspecific $(n=37)$, and control $(n=37)$ conditions and were tested individually in $\sim 25 \mathrm{~min}$ sessions.

Materials For the ongoing lexical decision task (LDT), following Lourenço et al. (2013), we selected 280 words from the ELP database (Balota et al., 2007) that were four to nine letters in length, consisted of two to four syllables, and had a mean log-transformed hyperspace analogue to language frequency of 8.0 (mean Kucera and Francis frequency of 82).
We also generated 296 nonwords from the ELP that were four to nine letters in length. All items were presented in lowercase, 30-point font and appeared in upper and lower portions of the screen. The upper location and lower location were presented $40 \%$ and $60 \%$, respectively, from the top of the screen. The size of the monitor was 19 in. with a resolution of $1280 \times$ 1024. The same PM targets were used as in Lourenço et al. (2013): dormitory, factory, history, torches, torment, tornado, tortoise, and victory.

Procedure The procedure was modeled after the blocked PM procedure used by Lourenço and Maylor (2014). As illustrated in Fig. 1, participants were first given instructions regarding the ongoing LDT for which they were to decide as quickly and accurately as possible if a string of letters was a word ( $F$ key) or a nonword ( $J$ key). Following each decision, a blank screen would appear, and participants were to press the space bar to progress to the next trial. Participants were also instructed that words and nonwords would appear in either the upper or lower location of the screen. Additionally, they were instructed that word type (word or nonword) and location (upper or lower) would be presented in blocks of eight trials (e.g., eight word trials would appear in the lower location followed by eight nonword trials in the lower location) throughout the experiment. The experimenter ensured that participants fully understood the LDT instructions as well as the blocking procedure prior to beginning a 16 trial practice phase.

As shown in Fig. 1, following practice all participants performed the baseline block. Upon completion of this block, all participants were told they would be continuing the LDT and reminded to perform the task as quickly and accurately as possible. Participants in the PM conditions (specific, nonspecific) were given additional instructions regarding the PM task prior to the PM block. They were told that in addition to the ongoing LDT task, whenever they saw the syllable "tor" they should press the 7 key after making their lexical decision or as soon as they remembered, even if the trial was no longer on the screen. In the specific condition, participants were told that the syllable "tor" would only occur in words in the upper location on the screen (and thus targets would never occur in nonwords or in the lower location). In the nonspecific condition, participants were told that the syllable could appear in either upper or lower locations and in words or nonwords. Participants were asked to summarize instructions and the experimenter checked that they understood the target syllable, the response key, and the target contexts (i.e., upper word trials only, or any combination of word type and location) before continuing. A 5-min delay following PM instructions was created by having the participants fill out a demographics questionnaire and complete the Shipley Vocabulary Test (Shipley, 1940). The procedure was the 


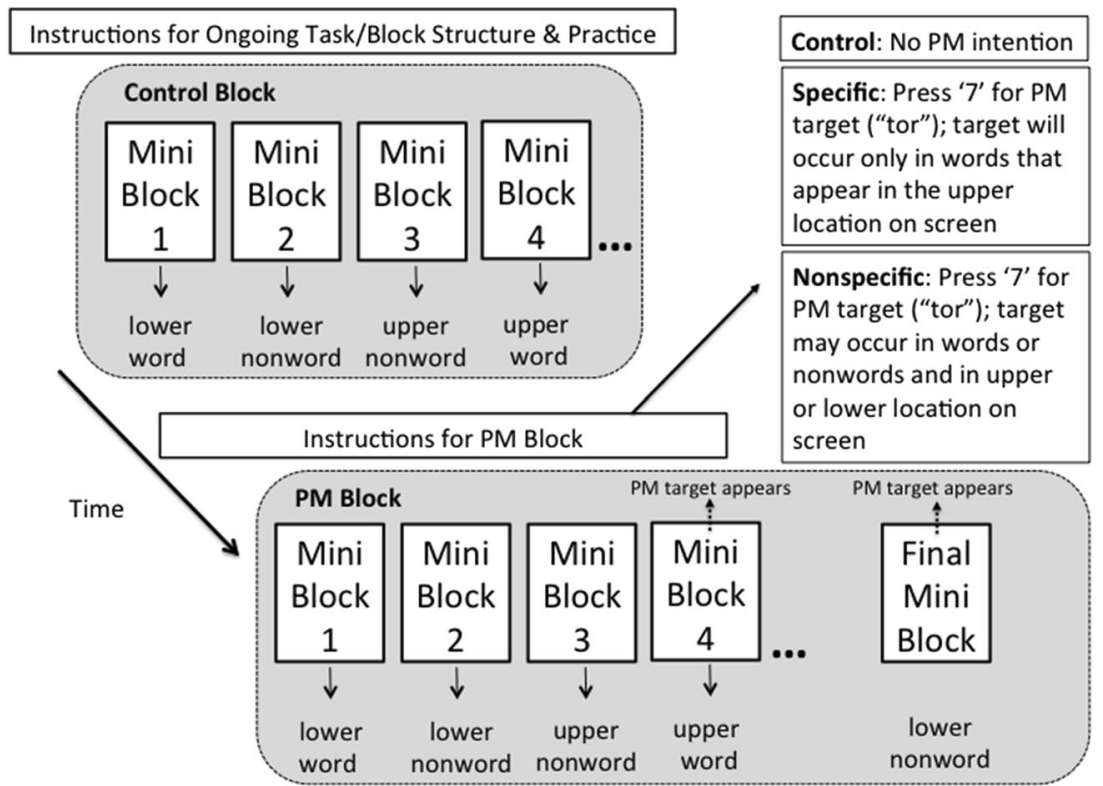

Fig. 1 Procedure used in Experiment 1. Each mini block comprised eight trials. Mini blocks were contiguous, meaning there was no break between mini blocks. The procedure was identical in Experiment 2, except that the specific condition was replaced with two probabilistic conditions, specific $_{-90}$ and specific -70 . In Experiment 3, the specific, specific ${ }_{-70}$, and nonspecific conditions were used. $\mathrm{PM}=$ prospective memory

block, which was an unexpected (lower nonword) context (see Fig. 1). At the end of the experiment, all participants filled out a postexperimental questionnaire to check their memory for the PM task.

Both the baseline and PM blocks were comprised of 256 LDT trials (128 words and 128 nonwords), with half of each presented in the upper location and half in the lower location. Within the baseline and PM blocks there were 32 eight-trial (mini) blocks (e.g., eight mini blocks for each context: upper words, lower nonwords; see Fig. 1). The order of these blocks was determined preexperimentally and was identical for each participant. The order was pseudorandomized such that no two blocks of the same context were presented consecutively (e.g., an upper nonword block could not follow an upper nonword block). Additionally, in the PM block, upper word blocks (in which PM targets appeared) always occurred as the fourth block in each eighth of the set of 256 trials. For both PM conditions (specific, nonspecific), the PM target syllable "tor" appeared in words in the upper location and was presented once in each of the eight upper word blocks. The PM targets were presented on trials 28, 62, 93, 125, 155, 190, 219 , and 252, and appeared between the third and sixth position of the eight trial blocks. The order of appearance for the eight PM targets was randomized between participants.

To examine PM target detection in the unexpected context, immediately following the end of the 256 trial PM block described above, we included three additional mini blocks consisting of eight trials each (one lower word block, one upper nonword block, and one lower nonword block, in that order). (To enable calculation of monitoring cost, the three additional mini blocks also appeared at the end of the baseline blocks.) Crucially, in the PM block a ninth PM target (torpidigy) was embedded on the fifth trial of the final mini

\section{Results}

Lexical decision task performance For accuracy and reaction time analyses, the first six trials of the baseline and PM blocks, the PM target trial, and the three trials immediately following the PM target were excluded to avoid potential bias related to the PM intention retrieval process. Additionally, the 24 trials from the three additional blocks (following the first 256 trials) in both the baseline and PM blocks were excluded from analyses. Reaction time analyses were only conducted on correct trials and were trimmed at 2.5 standard deviations from each participant's mean separately for each block (Lourenço et al., 2013).

Overall ongoing task accuracy was extremely high $(M=.96$, $S E=.03$ ), and there was little variation across blocks (baseline and PM) or conditions (specific, nonspecific, control). In fact, there was no evidence of cost due to possessing an intention $(F<1)$, and there was no interaction of block and condition with any other factors (i.e., word type or location), $F_{\mathrm{S}}<1.88$, $p \mathrm{~s}>.16$. Thus, for brevity, we do not report the full accuracy analyses and focus our results on reaction times below.

Mean RT (see Table 1) was submitted to a 2 (block: baseline vs. $P M) \times 2$ (word type: word vs. nonword $) \times 2$ (location: upper vs. lower) $\times 3$ (condition: control vs. specific vs. 
Table 1 Mean reaction times and costs (standard errors) across conditions in the expected and unexpected contexts in Experiments 1, 2, and 3

\begin{tabular}{|c|c|c|c|c|c|c|}
\hline \multirow[t]{2}{*}{ Experiment } & \multirow[t]{2}{*}{ Block } & \multirow[t]{2}{*}{ Condition } & \multirow{2}{*}{$\begin{array}{l}\text { Expected } \\
\text { Upper word }\end{array}$} & \multicolumn{3}{|l|}{ Unexpected } \\
\hline & & & & Lower word & Upper nonword & Lower nonword \\
\hline \multirow[t]{9}{*}{1} & \multirow[t]{3}{*}{ Baseline } & Control & $542(27)$ & $518(27)$ & 609 (37) & $613(37)$ \\
\hline & & Specific & $562(32)$ & $516(30)$ & $623(35)$ & $632(36)$ \\
\hline & & Nonspecific & $524(30)$ & $483(28)$ & $578(35)$ & $564(35)$ \\
\hline & \multirow[t]{3}{*}{ PM } & Control & $443(22)$ & 429 (29) & $442(31)$ & $408(30)$ \\
\hline & & Specific & $656(28)$ & $513(34)$ & $564(36)$ & $468(31)$ \\
\hline & & Nonspecific & $635(22)$ & $635(23)$ & $629(28)$ & $574(25)$ \\
\hline & \multirow[t]{3}{*}{ Cost } & Control & $-99(12)$ & $-89(11)$ & $-167(14)$ & $-205(13)$ \\
\hline & & Specific & $94(23)$ & $-3(18)$ & $-59(21)$ & $-164(20)$ \\
\hline & & Nonspecific & $111(25)$ & $152(20)$ & $51(25)$ & $10(27)$ \\
\hline \multirow[t]{12}{*}{2} & \multirow[t]{4}{*}{ Baseline } & Control & 433 (26) & 397 (27) & 478 (29) & 475 (29) \\
\hline & & Specific-90 & $498(31)$ & $456(28)$ & $563(36)$ & $533(31)$ \\
\hline & & Specific-70 & $336(22)$ & $311(21)$ & $369(25)$ & $373(22)$ \\
\hline & & Nonspecific & $508(32)$ & $476(29)$ & $597(50)$ & $594(47)$ \\
\hline & \multirow[t]{4}{*}{ PM } & Control & $353(22)$ & $312(26)$ & $322(27)$ & 299 (26) \\
\hline & & Specific-90 & $608(21)$ & $516(28)$ & $534(34)$ & 449 (28) \\
\hline & & Specific-70 & $524(23)$ & $452(28)$ & 464 (34) & $406(31)$ \\
\hline & & Nonspecific & $602(25)$ & $613(28)$ & $618(30)$ & $571(25)$ \\
\hline & \multirow[t]{4}{*}{ Cost } & Control & $-80(15)$ & $-85(10)$ & $-156(15)$ & $-175(18)$ \\
\hline & & Specific-90 & $109(25)$ & $60(21)$ & $-30(18)$ & $-83(19)$ \\
\hline & & Specific-70 & $188(22)$ & $141(23)$ & $95(28)$ & $32(26)$ \\
\hline & & Nonspecific & $94(28)$ & $137(24)$ & $21(34)$ & $-23(32)$ \\
\hline \multirow[t]{9}{*}{3} & \multirow[t]{3}{*}{ Baseline } & Specific & 564 (19) & $541(20)$ & $621(33)$ & $623(33)$ \\
\hline & & Specific-70 & $538(21)$ & $508(21)$ & $602(27)$ & 595 (28) \\
\hline & & Nonspecific & $558(24)$ & $529(22)$ & 627 (31) & $630(30)$ \\
\hline & \multirow[t]{3}{*}{ PM } & Specific & $665(25)$ & $525(24)$ & $579(30)$ & $472(25)$ \\
\hline & & Specific-70 & 644 (29) & $620(30)$ & $640(36)$ & $540(31)$ \\
\hline & & Nonspecific & 664 (24) & 676 (29) & $662(24)$ & $610(25)$ \\
\hline & \multirow[t]{3}{*}{ Cost } & Specific & $102(16)$ & $-16(12)$ & $-42(25)$ & $-151(22)$ \\
\hline & & Specific-70 & $106(21)$ & $112(18)$ & $38(23)$ & $-55(17)$ \\
\hline & & Nonspecific & $106(22)$ & 147 (22) & 35 (24) & $-20(23)$ \\
\hline
\end{tabular}

Note. The expected and unexpected columns refer to the contexts in which participants in the specific conditions expected or did not expect, respectively, PM targets to appear (Specific) or likely appear (Specific ${ }_{-90}$ and Specific -70 ). Participants in the nonspecific condition were told targets could appear in any context.

nonspecific) mixed-factorial analysis of variance (ANOVA). There was an effect of word type, $F(1,108)=9.35, p<.003$, $\eta^{2^{p}}=.08$, location, $F(1,108)=149.30, p<.001, \eta^{2 p}=.58$, and block, $F(1,108)=9.76, p=.002, \eta^{2}=.083$, with slower RTs for nonwords, in the upper location, and in the PM block. The effect of condition was not significant, $F(2,108)=2.21, p=$ $.115, \eta^{2}=.039$. However, other than the two-way interaction of word type and condition, $F(2,108)=1.16, p=.316, \eta^{2}=$ .021 , and a marginal four-way interaction, $F(2,108)=2.95, p$ $=.056, \eta^{2}=.052$, all higher order interactions were significant, Block $\times$ Word Type: $F(1,108)=214.55, p<.001, \eta^{2}=$ .665 ; Block $\times$ Location: $F(1,108)=43.47, p<.001, \eta^{2_{p}}=$ .287 ; Block $\times$ Condition: $F(2,108)=42.21, p<.001, \eta^{2}=$
439; Word Type $\times$ Location: $F(1,108)=5.46, p=.021, \eta^{2 p}=$ .048 ; Location $\times$ Condition: $F(2,108)=26.42, p<.001, \eta^{2}=$ 329; Block $\times$ Word Type $\times$ Location: $F(2,108)=12.98, p<$ $.001, \eta^{2}=.107$; Block $\times$ Word Type $\times$ Condition: $F(2,108)=$ 6.42, $p=.002, \eta^{2}=.106$; Block $\times$ Location $\times$ Condition: $F(2$, $108)=29.33, p<.001, \eta^{2 p}=.352$; Word Type $\times$ Location $\times$ Condition: $F(2,108)=11.58, p<.001, \eta^{2}=.177$. In subsequent analyses, we decompose the marginal four-way interaction of block, word type, location, and condition to examine whether participants showed evidence of strategic monitoring.

Of primary interest was comparing cost across conditions in expected and unexpected contexts (see Fig. 2). As a reminder, cost refers to the difference in ongoing task RT between the 


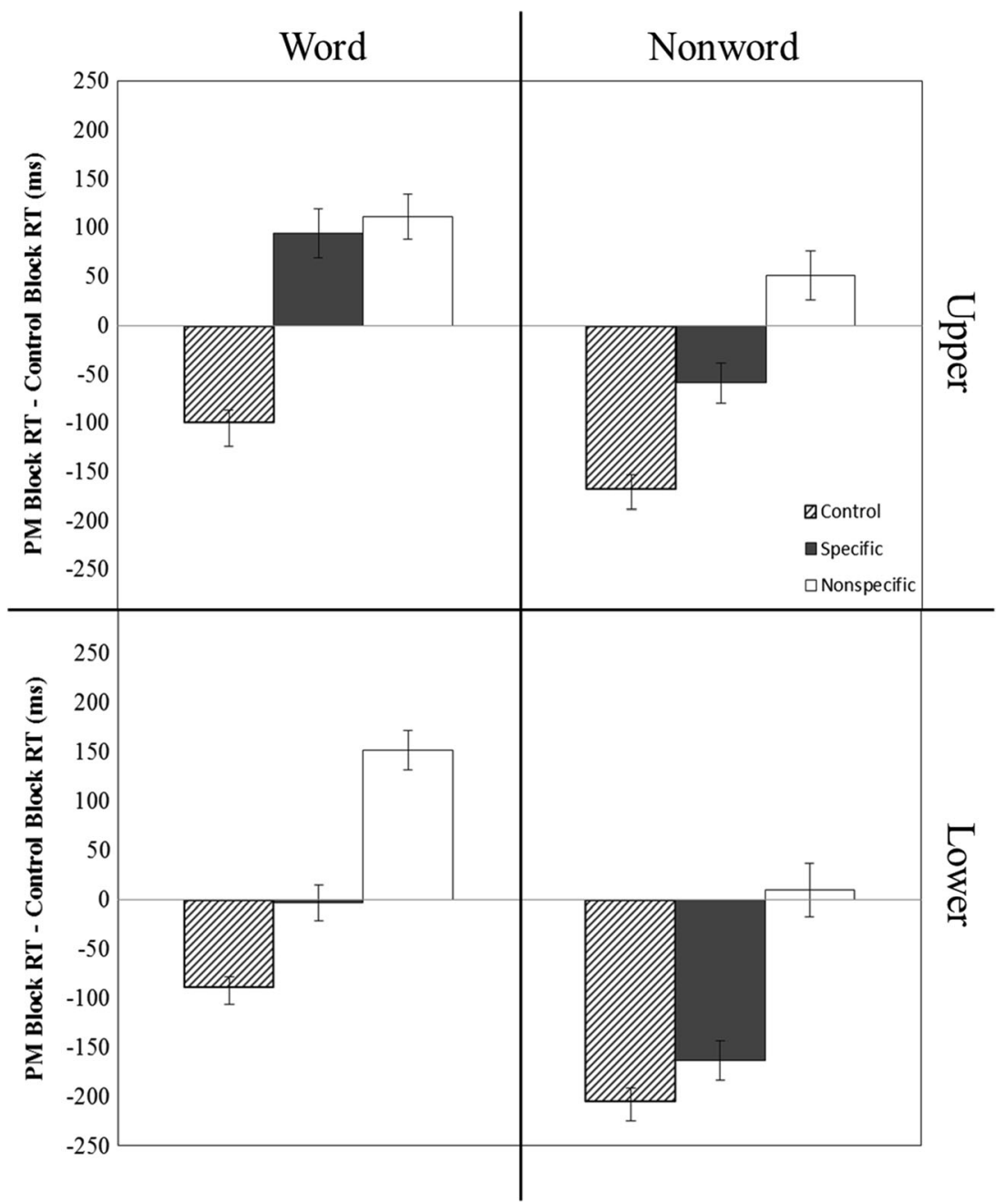

Fig. 2 Cost estimates plotted separately by word type (word, nonword) and location (upper, lower) for each condition in Experiment 1

baseline and PM blocks. Thus, we performed separate analyses to examine group differences across the four different contexts (i.e., upper word, upper nonword, lower word, lower nonword) by submitting mean RTs to a 2 (block: baseline vs. PM) $\times 3$ (condition: control vs. specific vs. nonspecific) mixed-factorial ANOVA.

Upper word (expected context) For word RTs in the upper location (i.e., where cues appeared), there was an effect of condition, $F(2,108)=5.88, p=.004, \eta^{2}=.098$, an effect of block, $F(1,108)=8.51, p=.004, \eta^{2}=.073$, and an interaction of block and condition, $F(2,108)=30.91, p<.001, \eta^{2 p}$ $=.364$. This interaction reflects that relative to the control condition ( $M=-99 \mathrm{~ms}, S E=12 \mathrm{~ms}$ ), cost was much greater in the nonspecific ( $M=111 \mathrm{~ms}, S E=25 \mathrm{~ms})$ and specific $(M=$ $94 \mathrm{~ms}, S E=23 \mathrm{~ms})$ conditions, $F(1,72)=56.02, p<.001, \eta^{2 p}$ $=.438$, and $F(1,72)=55.33, p<.001, \eta^{2}=.435$, respectively. However, there were no cost differences between the nonspecific and specific conditions, $F<1$.
Lower word (unexpected context) For word RTs in the lower location, there was no effect of condition, $F(2,108)=2.46$, $p=.09, \eta^{2}=.044$. However, there was an effect of block, $F(1$, $108)=4.25, p<.001, \eta^{2 p}=.038$, and an interaction of block and condition, $F(2,108)=55.87, p<.001, \eta^{2}=.499$. This interaction reflects that relative to the control condition $(M=$ $89 \mathrm{~ms}, S E=11 \mathrm{~ms}$ ), cost was much greater in the nonspecific $(M=152 \mathrm{~ms}, S E=20 \mathrm{~ms})$ and specific $(M=-3 \mathrm{~ms}, S E=$ $18 \mathrm{~ms}$ ) conditions, $F(1,72)=111.28, p<.001, \eta^{2}=.607$, and $F(1,72)=17.50, p<.001, \eta^{2 p}=.196$, respectively. ${ }^{1}$ Additionally, cost was greater for the nonspecific than specific condition, $F(1,72)=33.47, p<.001, \eta^{2 p}=.317$.

Upper nonword (unexpected context) For nonword RTs in the upper location, there was no effect of condition, $F(2,108)$

\footnotetext{
${ }^{1}$ Although cost was a negative value $(-3 \mathrm{~ms})$ in the specific condition, this is still considered a monitoring cost because relative to the control condition (no PM intention) that demonstrated a $-89-\mathrm{ms}$ cost, the speed up from the control to the PM block was less robust (likely due to PM monitoring demands).
} 
$=1.77, p=.176, \eta^{2}=.032$. However, there was an effect of block, $F(1,108)=23.91, p<.001, \eta^{2 p}=.181$, and an interaction of block and condition, $F(2,108)=28.11, p<.001, \eta^{2 p}$ $=.342$. This interaction reflects that relative to the control condition $(M=-167 \mathrm{~ms}, S E=14 \mathrm{~ms})$, cost was much greater in the nonspecific $(M=51 \mathrm{~ms}, S E=25 \mathrm{~ms})$ and specific $(M=-$ $59 \mathrm{~ms}, S E=21 \mathrm{~ms})$ conditions, $F(1,72)=56.51, p<.001, \eta^{2 p}$ $=.440$, and $F(1,72)=18.61, p<.001, \eta^{2 p}=.205$, respectively. Additionally, cost was greater for the nonspecific than specific condition, $F(1,72)=11.30, p=.001, \eta^{2 p}=.136$.

Lower nonword (unexpected context) For nonword RTs in the lower location, there was no effect of condition, $F<1$. However, there was an effect of block, $F(1,108)=100.22, p<$ $.001, \eta^{2 p}=.481$, and an interaction of block and condition, $F(2,108)=30.24, p<.001, \eta^{2}=.359$. This interaction reflects that relative to the control condition $(M=-205 \mathrm{~ms}, S E=$ $13 \mathrm{~ms})$, cost was much greater in the nonspecific $(M=10 \mathrm{~ms}$, $S E=27 \mathrm{~ms})$ condition, $F(1,72)=51.72, p<.001, \eta^{2}=.418$. Additionally, cost was greater for the nonspecific than specific ( $M=-164 \mathrm{~ms}, S E=20 \mathrm{~ms}$ ) condition, $F(1,72)=26.68, p<$ $.001, \eta^{2}=.270$. However, there was not a difference between the control and specific condition, $F(1,72)=3.10, p=.083$, $\eta^{2 p}=.041$.

PM target detection in expected context To examine PM performance, the proportion of successfully detected PM targets within the upper word context was submitted to a between-subjects ANOVA (see left portion of Fig. 3). This analysis revealed no differences in target detection between specific $(M=.89, S E=.03)$ and nonspecific $(M=.90, S E=$ 03) conditions, $F<1$.

PM target detection in unexpected context To examine PM target detection in the unexpected context, the number of participants that detected the PM target in the final (lower nonword) block was submitted to a chi-square test (left-hand portion of Fig. 4). This analysis revealed that more participants detected the PM target in the nonspecific condition (76\%) than in the specific (5\%) condition, $\chi^{2}(1, N=74)=37.90, p<.001$.

We also examined whether monitoring cost (PM block control block RTs) in the lower nonword context was predictive of target detection (collapsed across conditions). As expected, a logistic regression revealed that individuals that exhibited greater cost were more likely to detect the final target in the unexpected context, Wald $\chi^{2}(1, N=74)=17.30, p<.001$.

\section{Discussion}

The primary purpose of Experiment 1 was to examine whether strategic monitoring is evidenced in response to a complex contextual cue. This question was addressed by means of the blocked PM procedure that places lower demands on attentional resources in service of identifying a contextual cue and allocating attention accordingly (relative to a random PM procedure; Lourenço \& Maylor, 2014). The key finding was that strategic monitoring was observed in response to the conjunction represented by the complex contextual cue. Participants in the specific and nonspecific conditions showed equivalent cost in the upper word context, whereas in all other contexts (upper nonword, lower word, lower nonword) cost was reduced for participants in the specific relative to the nonspecific condition. This indicates that participants in the specific condition engaged monitoring similarly to those in the nonspecific condition in contexts in which they expected PM targets to appear and successfully relaxed monitoring in contexts in which they did not expect PM targets. Neatly, this provides initial evidence for strategic monitoring in response to a complex contextual cue.

As is routinely the case, it was also found that cost was overall greater for both the nonspecific and specific conditions relative to the control condition that performed merely the ongoing task in the second block of trials. More relevant to the present goals, the comparison of cost between the specific condition and the control condition in the unexpected contexts generally suggested that cost was not eliminated for participants in the specific condition (i.e., there was more cost in the specific than in the control). This coincides with findings from the blocked PM condition of Lourenço and Maylor (2014). They reasoned cost was not eliminated because the intention was kept at a sufficiently high level of activation to facilitate response execution when the cued context was encountered (i.e., the prospective retrieval mode was maintained to some degree across contexts). There was one exception to this pattern in the present experiment - cost was eliminated for the specific condition in the lower nonword context (although the difference in cost between the specific and control conditions trended towards significance). Because this finding is at odds with Lourenço and Maylor as well as Guynn's (2003) model, which posits that cost should never be completely eliminated (i.e., the presence of the retrieval mode should always incur some cost; but see Cohen et al., 2012), and we did not observe an elimination of cost in a follow-up study, ${ }^{2}$ we hesitate to draw any firm theoretical conclusions regarding this pattern.

The results from Experiment 1 additionally provided evidence for a potential downfall to strategic monitoring, namely,

\footnotetext{
${ }^{2}$ As part of another study, we collected data from specific $(N=30)$ and nonspecific $(N=30)$ conditions using identical methodology as reported here (without the ninth PM target). Similar results were found comparing the specific and nonspecific conditions, replicating the pattern of strategic monitoring for complex contextual cues in the specific condition. However, when we compare the specific condition from that study to the control condition of Experiment 1, there is a significant difference in cost in the lower-nonword context $\left(M_{\text {specific }}=-145, S E=21 ; M_{\text {control }}=-205, S E=13\right), F(1,65)=6.60, p$ $=.012, \eta^{2}=.092$, suggesting cost was not eliminated in the specific condition.
} 

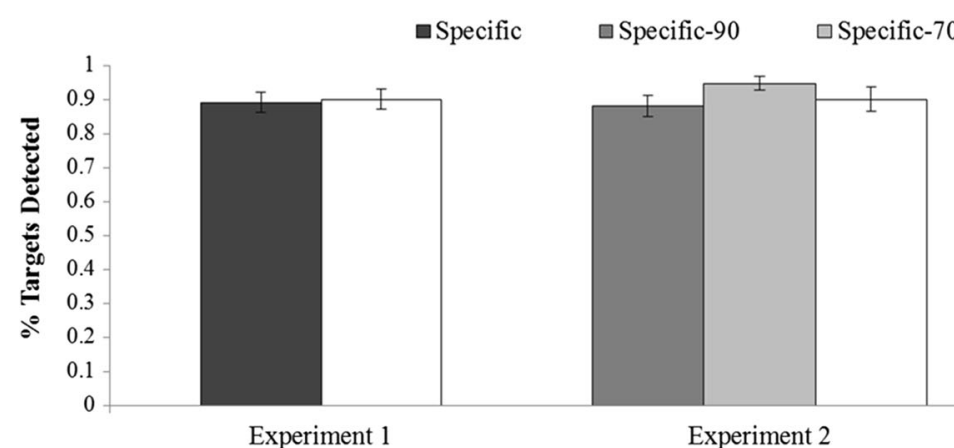

$\square$ Nonspecific

Fig. 3 Proportion of PM targets detected across conditions in the expected context for each experiment. "Expected" refers to the context (i.e., upper word) in which participants in the specific conditions expected
PM targets to appear (specific) or likely appear (specific- 90 and specific 70). Participants in the nonspecific condition were told targets could appear in any context). Error bars reflect standard errors a reduction in PM target detection in the unexpected, lower nonword context for participants in the specific relative to the nonspecific condition. This predicted pattern rested on the assumption that the specific condition would relax monitoring in the unexpected contexts, including in the lower nonword context. This assumption was confirmed, and relaxation of monitoring was nominally greatest in the lower nonword context. Most critically, detection of the PM target was significantly less likely for participants in the specific condition. Only $5 \%$ of participants detected the target compared to $76 \%$ of participants in the nonspecific condition. Moreover, across both conditions, larger cost in the lower nonword context (indicative of more monitoring) was associated with a greater likelihood of PM target detection. These findings converge with and extend the findings of Cook et al. (2005) who used a time-based PM task. They demonstrated reduced PM performance when the window for responding occurred within a time frame that differed from the expected one (i.e., occurred in an unexpected context).

Finally, we note that consistent with prior research (Kuhlmann \& Rummel, 2014; Lourenço \& Maylor, 2014; Lourenço et al., 2013), PM performance did not differ across specific and nonspecific conditions. This is logical because
PM targets for the specific and nonspecific conditions always occurred in the upper word context that served as an "expected" context for both conditions and monitoring was statistically equivalent in the upper word context for participants in the specific and nonspecific conditions.

\section{Experiment 2}

Using a variant of the blocked PM procedure (Lourenço \& Maylor, 2014), Experiment 1 indicated that strategic monitoring may be observed when the cue signaling an intentionrelevant context is complex rather than simple. In Experiment 2, we thought it valuable to investigate whether the expected value of control, which has been shown to influence decisions individuals make about whether to utilize costly capacity-limited cognitive resources (Shenhav, Botvinick, $\&$ Cohen, 2013), affects the robustness of the strategic monitoring pattern given its dependence on such resources (e.g., Lourenço \& Maylor, 2014). We achieved this aim by investigating another potential boundary condition for strategic monitoring - use of a probabilistic contextual cue, a type of cue that has not yet been examined in this literature but may be

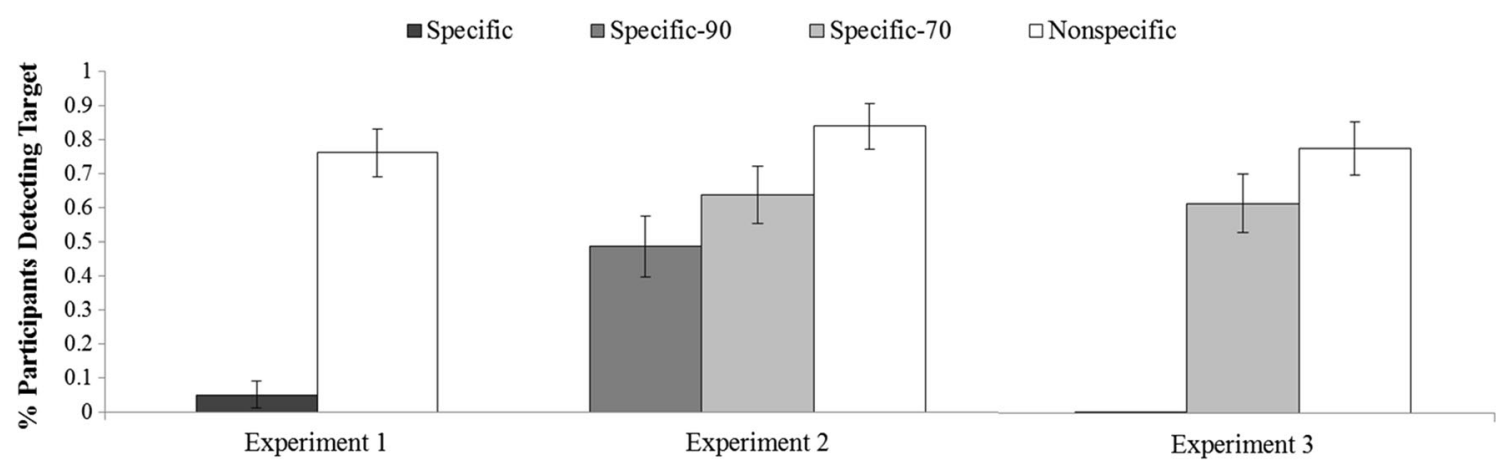

Fig. 4 Percentage of participants across conditions detecting the PM target in the unexpected context for each experiment. "Unexpected" refers to a context (lower nonword) in which participants in the specific conditions did not expect (specific) or did not likely expect (specific-90 and specific $_{-70}$ ) PM targets to appear. Participants in the nonspecific

condition were told targets could appear in any context. Note that in Experiment 3, the left bar refers to the specific condition, and $0 \%$ of participants detected the target in that condition. Error bars reflect standard errors 
common in the real world. Extending the earlier example, it may be probable though not certain that one's professor (to whom a message must be delivered) would be encountered in the building that houses her office, but not in the student recreation center. The question is whether this type of advance information (expectation) would still be used to monitor strategically. Experiment 2 was identical to Experiment 1, except that there were two specific conditions-participants were told either that there was a $90 \%$ likelihood of PM targets occurring in the upper word context and that cues would almost never appear in other contexts (specific -90 condition) or they were told there was a 70\% likelihood of PM targets occurring in the upper word context and cues would usually not appear in other contexts (specific -70 condition).

The rationale was as follows. If strategic monitoring depends on costly attentional processes, then decisions to engage strategic monitoring may be influenced by required effort and expected reward (i.e., expected value of control; Shenhav et al., 2013). That is, the expected reward (detecting PM targets and conserving resources in unexpected contexts) may not be sufficiently high to justify the effort (attentional resources) required to identify the contextual cue and modulate monitoring in a context-specific fashion in the specific $_{-70}$ condition or even the specific -90 condition, nor may it justify the potential cost of missing out on PM targets in unexpected contexts. If so, strategic monitoring for a complex probabilistic cue may not be evidenced. In line with this prediction, Lourenço and Maylor (2014) found that strategic monitoring was weakened by the occasional presence of a PM target in an unexpected context, and this may be because the occurrence of these targets decreased the validity of the contextual cue. An alternative prediction was that strategic monitoring would be observed in spite of the contextual uncertainty associated with the probabilistic cue. Such a pattern would suggest that use of probabilistic cues is not a boundary condition for strategic monitoring and would also have important theoretical and practical implications.

\section{Method}

Design and participants The design was a $4 \times 2 \times 2 \times 2$ mixed-factorial, with condition (specific- 90 , specific- 70 , nonspecific, control) as a between-subjects factor and block (baseline, PM), word type (word, nonword), and location (upper, lower) as within-subjects factors. Participants ages 18 to 25 years were undergraduates at Washington University in St. Louis, taken from the psychology subject pool. One hundred and twenty-eight participants were randomly assigned to the specific- ${ }_{90}(n=33)$, specific- ${ }_{70}(n=33)$, nonspecific $(n=$ $31)$, or control $(n=31)$ condition and were tested individually in $\sim 25$-min sessions.
Materials The materials were identical to those used in Experiment 1.

Procedure The procedure was identical to Experiment 1 for the control and nonspecific conditions (see Fig. 1). In Experiment 2, participants in the specific-90 condition were told that there was a $90 \%$ likelihood that the "tor" syllable would appear within words in the upper location, and therefore the "tor" syllable would almost never appear within a nonword or in the lower location. Participants in the specific- -70 condition were told that there was a $70 \%$ likelihood that the "tor" syllable would appear within words in the upper location, and therefore the "tor" syllable would usually not appear within a nonword or in the lower location. In all other regards, Experiment 2 was identical to Experiment 1, including the fact that the PM target was presented only in the upper word context for all conditions, with the exception of the last target that was presented to examine cue detection in an unexpected (lower nonword) context.

\section{Results}

Lexical decision task performance Trimming procedures for accuracy and RT analyses were identical to those of Experiment 1 . As with Experiment 1, overall ongoing task accuracy was extremely high $(M=.96, S E=.03)$, and there was little variation across blocks (baseline and PM) or conditions (specific, nonspecific, control). Again, there was no evidence of cost due to possessing an intention $(F<1.9)$, and there was no interaction of block and condition with any other factors (i.e., word type or location), $F \mathrm{~s}<1.22, p \mathrm{~s}>.303$. Thus, for brevity we do not report the full analyses and focus our results on reaction times below.

Mean RT (see Table 1) was submitted to a 2 (block: baseline vs. $\mathrm{PM}) \times 2$ (word type: word vs. nonword) $\times 2$ (location: upper vs. lower) $\times 4$ (condition: specific-90 vs. specific-70 vs. nonspecific vs. control) mixed-factorial ANOVA. There was an effect of word type, $F(1,124)=9.54, p=.003, \eta^{2_{p}}=.071$, location, $F(1,124)=165.87, p<.001, \eta^{2 p}=.572$, and condition, $F(3,124)=11.88, p<.001, \eta^{2}=.223$, with slower RTs for words, in the upper location, and for participants in the PM conditions. The effect of block was not significant, $F(1,124)=$ $2.22, p=.139$. However, other than a marginal three-way interaction of block, word type, and condition, all other higher order interactions including the four-way interaction were significant, Block $\times$ Word Type: $F(1,124)=199.92, p<.001, \eta^{2 p}$ $=.617$; Block $\times$ Location: $F(1,124)=32.30, p<.001, \eta^{2 p}=$ .207 ; Block $\times$ Condition: $F(3,124)=24.07, p<.001, \eta^{2 p}=$ .368 ; Word Type $\times$ Location: $F(1,124)=4.10, p=.045, \eta^{2 p}=$ .032 ; Word Type $\times$ Condition: $F(3,124)=4.64, p=.004, \eta^{2 p}=$ .101 ; Location $\times$ Condition: $F(3,124)=11.98, p<.001, \eta^{2 p}=$ 
.225 ; Block $\times$ Word Type $\times$ Location: $F(1,124)=9.18, p=.003$, $\eta^{2}=.069$; Block $\times$ Word Type $\times$ Condition: $F(3,124)=2.51, p$ $=.062, \eta^{2}=.057$; Block $\times$ Location $\times$ Condition: $F(3,124)=$ $6.84, p<.001, \eta^{2}=.142$; Word Type $\times$ Location $\times$ Condition: $F(3,124)=3.03, p=.032, \eta^{2}=.068$; Block $\times$ Word Type $\times$ Location $\times$ Condition: $F(3,124)=3.52, p=.017, \eta^{2}=.078$. In subsequent analyses, we decompose the four-way interaction to examine whether participants were sensitive to task instructions in their monitoring of PM targets.

Of primary interest was comparing cost across expected and unexpected contexts for the various conditions (see Fig. 5). Thus, we performed separate analyses for each of the four different contexts (i.e., upper word, upper nonword, lower word, lower nonword) by submitting mean RTs to a 2 (block: baseline vs. PM) $\times 4$ (condition: specific- 90 vs. specific-70 vs. nonspecific vs. control) mixed-factorial ANOVA.

Upper word (expected context) For word RTs in the upper location, there was an effect of block, $F(1,124)=45.97, p<$ $.001, \eta^{2}=.270$, an effect of condition, $F(3,124)=13.54, p<$
$.001, \eta^{2 p}=.247$, and an interaction of block and condition, $F(3,124)=24.04, p<.001, \eta^{2}=.368$. This interaction reflects that relative to the control condition $(M=-80 \mathrm{~ms}, S E=$ $15 \mathrm{~ms})$, cost was much greater in the nonspecific condition ( $M$ $=94 \mathrm{~ms}, S E=28 \mathrm{~ms}), F(1,60)=30.38, p<.001, \eta^{2 p}=.336$, the specific-90 condition ( $M=109 \mathrm{~ms}, S E=25 \mathrm{~ms}), F(1,62)=$ $40.42, p<.001, \eta^{2 p}=.395$, and the specific-70 condition $(M=$ $188 \mathrm{~ms}, S E=22 \mathrm{~ms}), F(1,62)=101.16, p<.001, \eta^{2 p}=.620$. However, while there was no cost difference between the nonspecific and specific-90 conditions, $F<1$, participants in the specific- -70 condition actually exhibited more cost than those in the specific- 90 and nonspecific conditions, $F(1,64)=5.66$, $p=.02, \eta^{2_{p}}=.081$, and $F(1,62)=7.24, p=.009, \eta^{2 p}=.105$, respectively. Thus, while all PM groups experienced cost relative to the control group, those in the specific-70 condition actually exhibited more cost than the other two groups.

Lower word (unexpected context) For word RTs in the lower location, there was an effect of block, $F(1,124)=37.71, p<$ $.001, \eta^{2 p}=.233$, an effect of condition, $F(3,124)=12.66, p<$

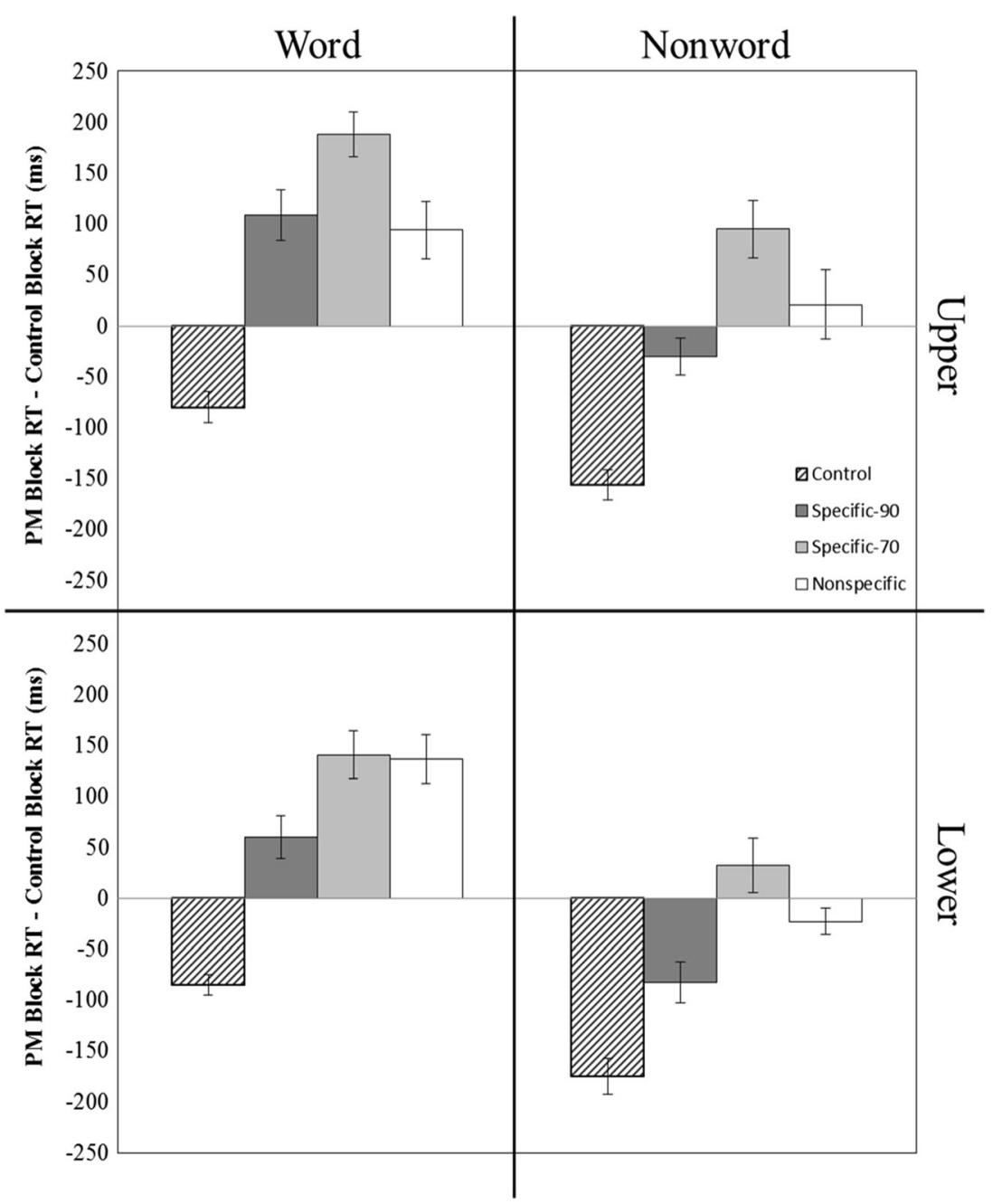

Fig. 5 Cost estimates plotted separately by word type (word, nonword) and location (upper, lower) for each condition in Experiment 2 
$.001, \eta^{2}=.235$, and an interaction of block and condition, $F(3$, 124) $=25.76, p<.001, \eta^{2}=.384$. This interaction reflects that relative to the control condition $(M=-85, S E=10)$, cost was much greater in the nonspecific condition $(M=137 \mathrm{~ms}, S E=$ $24 \mathrm{~ms}), F(1,60)=72.51, p<.001, \eta^{2}=.547$, the specific- 90 condition $(M=60 \mathrm{~ms}, S E=22 \mathrm{~ms}), F(1,62)=36.48, p<.001$, $\eta^{2^{p}}=.370$, and the specific -70 condition $(M=141 \mathrm{~ms}, S E=$ $23 \mathrm{~ms}), F(1,62)=77.57, p<.001, \eta^{2}=.556$. Additionally, while there were no cost differences between nonspecific and specific- 70 conditions, $F<1$, there was greater cost in the nonspecific than specific-90 condition, $F(1,62)=5.62, p=.021, \eta^{2}=.083$, and greater cost in the specific- 70 condition than the specific- 90 condition, $F(1,64)=6.49, p=.013, \eta^{2}=.092$. Thus, while all PM groups experienced cost relative to the control group, those in the specific- 90 condition were able to reduce monitoring relative to the specific-70 and nonspecific conditions.

Upper nonword (unexpected context) For nonword RTs in the upper location, there was no effect of block, $F=1.93, p=$ $.167, \eta^{2}=.135$. However, there was an effect of condition, $F(3,124)=10.16, p<.001, \eta^{2}=.197$, and an interaction of block and condition, $F(3,124)=18.01, p<.001, \eta^{2}=.303$. This interaction reflects that relative to the control condition $(M=-156 \mathrm{~ms}, S E=15 \mathrm{~ms})$, cost was much greater in the nonspecific condition $(M=21 \mathrm{~ms}, S E=34 \mathrm{~ms}), F(1,60)=$ $22.98, p<.001, \eta^{2}=.277$, the specific- 90 condition $(M=$ $30 \mathrm{~ms}, S E=18 \mathrm{~ms}), F(1,62)=27.91, p<.001, \eta^{2_{p}}=.310$, and the specific-70 condition ( $M=95 \mathrm{~ms}, S E=28 \mathrm{~ms}), F(1,62)=$ $60.33, p<.001, \eta^{2}=.493$. Furthermore, participants in the specific-70 condition exhibited more cost than those in the specific-90 condition, $F(1,64)=14.03, p<.001, \eta^{2}=.180$. However, there were no cost differences between the specific70 and nonspecific conditions, $F(1,62)=2.89, p=.094, \eta^{2}=$ .029 , or between the specific- 90 and nonspecific conditions, $F(1,62)=1.82, p=.182, \eta^{2 p}=.029$. Thus, while all PM groups experienced cost relative to the control group, both specific conditions exhibited similar cost to the nonspecific condition (though they actually differed from each other).

Lower nonword (unexpected context) For nonword RTs in the lower location, there was an effect of block, $F(1,124)=26.62$, $p<.001, \eta^{2}=.177$, an effect of condition, $F(3,124)=11.08, p<$ $.001, \eta^{2}=.211$, and an interaction of block and condition, $F(3$, 124) $=13.49, p<.001, \eta^{2}=.246$. This interaction reflects that relative to the control condition $(M=-175 \mathrm{~ms}, S E=18 \mathrm{~ms})$, cost was much greater in the nonspecific condition $(M=-23 \mathrm{~ms}, S E=$ $32 \mathrm{~ms}), F(1,60)=17.31, p<.001, \eta^{2}=.224$, the specific- 90 condition $(M=-83 \mathrm{~ms}, S E=19 \mathrm{~ms}), F(1,62)=12.59, p=.001$, $\eta^{2 p}=.169$, and the specific- ${ }_{70}$ condition $(M=32 \mathrm{~ms}, S E=26 \mathrm{~ms})$, $F(1,62)=42.65, p<.001, \eta^{2}=.408$. However, while there were no cost differences between nonspecific and specific-90 conditions, $F(1,62)=2.75, p=.102, \eta^{2}=.042$, or between nonspecific and specific-70 conditions, $F(1,62)=1.85, p=$ $.179, \eta^{2}=.029$, participants in the specific-70 condition exhibited more cost than those in the specific- 90 condition, $F(1,64)=13.27$, $p=.001, \eta^{2}=.172$. Thus, while all PM groups experienced cost relative to the control group, both specific conditions exhibited similar cost to the nonspecific condition (though they actually differed from each other).

PM target detection in expected context To examine PM performance, the proportion of successfully detected PM targets within the upper word context was submitted to a between-subjects ANOVA (middle portion of Fig. 3). This analysis revealed no differences in target detection between specific- $90(M=.88, S E=.03)$, specific-70 $(M=.95, S E=.03)$, and nonspecific $(M=.90, S E=.04)$ conditions, $F<1$.

PM target detection in unexpected context To examine PM target detection in the unexpected context, the number of participants that detected the PM target in the final (lower nonword) block was submitted to a chi-square test (middle portion of Fig. 4). This analysis revealed a significant difference in the number of participants that detected the target across conditions, $\chi^{2}(1, N=97)=8.83, p=.013$. Fewer participants in the specific- 90 condition (49\%) detected the PM target than in the nonspecific condition $(84 \%), \chi^{2}(1, N=64)=8.87, p=.003$, but an equal number of participants detected the target in the specific- 90 condition and the specific-70 condition $(64 \%)$, $\chi^{2}(1, N=66)=1.54, p=.215$. Additionally, there was only a marginal difference between nonspecific and specific- 70 conditions, $\chi^{2}(1, N=64)=3.36, p=.067$.

We also examined whether monitoring cost (PM block - control block RTs) in the lower nonword context was predictive of target detection (collapsed across conditions). As expected, a logistic regression revealed that individuals that exhibited greater cost were more likely to detect the final target in the unexpected context, Wald $\chi^{2}(1, N=97)=4.28, p=.039$.

\section{Discussion}

The primary aim of Experiment 2 was to examine whether strategic monitoring is evidenced in response to a complex contextual cue that is probabilistic. In the strategic monitoring literature to date, including the preceding experiments, contextual cues have always been deterministic - participants in the specific condition were told cues would appear only in a particular context (e.g., Kuhlmann \& Rummel, 2014; Lourenço \& Maylor, 2014; Lourenco et al., 2013; Marsh et al., 2006). In the specific conditions in the present study, participants were told there was a $90 \%$ or $70 \%$ likelihood cues would appear in the upper word context, and cues would almost never appear or would usually not appear, 
respectively, in the other contexts. Although there was a four-way interaction between block, condition, location, and word type, the evidence for strategic monitoring was not nearly as definitive as in Experiment 1, wherein a deterministic contextual cue was used. In the expected (upper word) context, the specific- 90 and specific70 conditions exhibited as much monitoring as the nonspecific condition (and monitoring was actually heightened in the specific- ${ }_{-70}$ condition). However, in the unexpected contexts, the specific-70 condition monitored as much as the nonspecific condition (i.e., specific-70 condition did not relax monitoring). A slightly different pattern was observed for the specific- 90 condition such that, relative to the nonspecific condition, monitoring was relaxed, but this pattern was found only in one of the three unexpected contexts (the lower word context). Had this pattern additionally been found in the lower nonword context it might suggest the location dimension of the complex cue (relatively automatically) triggered a relaxation of monitoring (i.e., turning off of retrieval mode), in spite of participants' decision not to strategically monitor given the probabilistic nature of the cue. However, at this point it appears random. Finally, contrasting monitoring patterns across the two specific conditions indicated that monitoring was greater for all contexts for the specific-70 condition, except for the lower word context. Together, these findings suggest that increasing contextual uncertainty via use of probabilistic cues appears to limit use of strategic monitoring (cf. Bugg \& Smallwood, 2016, for evidence that use of preparatory attentional resources is deterred by probabilistic cues in a different type of task). This fits with the assumption that strategic monitoring depends on costly, limited-capacity attentional resources (e.g., Lourenço \& Maylor, 2014; Lourenço et al., 2013), and therefore, like other costly cognitive processes, an individuals' willingness to engage strategic monitoring may be influenced by the required effort and expected reward associated with its use (e.g., Shenhav et al., 2013).

A second question we addressed concerned a potential disadvantage associated with monitoring strategically — namely, the failure to detect a PM target that occurs in an unexpected context. Considering the above patterns demonstrating weak evidence for strategic monitoring in the specific- 90 and specific- ${ }_{70}$ conditions, and in particular the fact that the specific- -90 and specific-70 conditions showed levels of monitoring equivalent to the nonspecific condition in the lower nonword context in which the PM target was presented, it was reasonable to expect that PM target detection would be equivalent across the three conditions. However, the findings were more variable. A significantly greater number of participants detected the PM target in the nonspecific condition relative to the specific-90 condition, while the specific-70 condition fell in the middle and did not differ from either the nonspecific or the specific-90 condition.

Finally, as with Experiment 1, there was no difference in PM target detection across the three PM conditions in the expected context. Again, that is logical because monitoring was as high (specific-90) or even higher (specific-70) in the specific conditions as the nonspecific condition.

\section{Experiment 3}

The findings of Experiments 1 and 2 generally support the view that strategic monitoring in response to a complex but probabilistic contextual cue is less robust than strategic monitoring in response to a complex contextual cue that is deterministic. However, the question of whether probabilistic cues truly limit the use of strategic monitoring processes merits further examination because of the preliminary nature of Experiment 2 (i.e., it was the first experiment to investigate strategic monitoring in response to probabilistic cues) and the dependence of the above conclusion on a cross-experimental comparison of strategic monitoring patterns (i.e., comparison of specific condition in Experiment 1 to specific- 90 and specific- -70 conditions of Experiment 2). To address these limitations, we randomly assigned participants to a specific (deterministic), specific70 (probabilistic), or nonspecific condition in Experiment 3 and contrasted patterns of strategic monitoring. ${ }^{3}$

A second aim was to address another limitation of the preceding experiments. Recall that we examined the proportion of participants who responded to the PM target in the unexpected context to evaluate the question of whether strategic monitoring (in the specific condition) may be accompanied by a disadvantage to performance. Experiment 1 demonstrated that dramatically fewer participants in the specific condition detected the target compared to the nonspecific condition, suggesting a disadvantage, whereas the target detection patterns in Experiment 2 were less definitive. A concern with the approach we adopted, however, is that it is uncertain whether the absence of a response to the PM target in the unexpected context constitutes a true "miss" or if participants noticed the PM target but thought the instructions implied they should respond to targets in the upper word context only. The latter possibility is especially relevant to the interpretation of participants' performance in the specific condition because these participants did not expect PM targets to occur outside of the upper word context. If participants noticed the target but chose not to respond because they thought they should not, then Experiment 1 may have overestimated the disadvantage associated with strategic monitoring. To address this concern, we modified the procedure in the final (lower nonword) block such that after the PM target was presented in the unexpected context, participants were explicitly asked if they noticed the PM target. They were also asked to indicate whether or not they responded to the target and, in the case they did not respond, indicate why. This allowed us to examine whether the very low rate of target detection in the unexpected context

\footnotetext{
${ }^{3}$ We did not include a control condition that never possessed a PM intention in this experiment because it was not necessary for addressing the primary question of interest concerning patterns of strategic monitoring across deterministic and probabilistic specific conditions and the nonspecific condition.
} 
for the specific condition was due to a true disadvantage of engaging in strategic monitoring or the alternative explanation.

\section{Method}

Design and participants The design was a $3 \times 2 \times 2 \times 2$ mixed-factorial, with condition (specific, nonspecific, specific-70) as a between-subjects factor, and block (baseline, PM), word type (word, nonword), and location (upper, lower) as within-subjects factors. Participants ages 18 to 25 years were undergraduates at Washington University in St. Louis, taken from the psychology subject pool. One hundred and three participants were randomly assigned to the specific $(n=35)$, nonspecific $(n=33)$, or specific- $70(n=35)$ condition and were individually tested in $\sim 25$ min sessions.

Materials and procedure The materials and procedure were identical to those of Experiment 1 for the specific and nonspecific conditions, and Experiment 2 for the specific-70 condition, with one exception: Immediately following the end of the lexical decision task in the PM block, participants were queried about their memory for the occurrence of the unexpected PM target (the target that was presented in the immediately preceding lower nonword context). Participants were first asked, "Was an item with the 'tor' syllable presented within the last five trials of the previous task?" (The target actually appeared four trials prior). If a participant responded "no," then the computer exited out of the program. If a participant responded "yes," then participants were asked, "When you saw the 'tor' syllable at the end of the task, did you respond with the '7' key?" If responded to with "yes," then the computer exited out of the program. If responded to with "no," then participants were asked, "Why did you not press the ' 7 ', key when you saw the 'tor' syllable at the end of the task?" After the participants typed in their response, the computer exited out of the program.

\section{Results}

Lexical decision task performance Trimming procedures for accuracy and RT analyses were identical to those of the previous experiments. As with the previous experiments, overall ongoing task accuracy was extremely high $(M=.95, S E=$ .03 ), and there was little variation across blocks (baseline and PM) or conditions (specific, nonspecific, specific-70). Although there was a hint of cost due to possessing an intention, $F(1,100)=3.50, p=.06, \eta^{2}=.034$, there was no interaction of block and condition with any other factors (i.e., word type or location), $F \mathrm{~s}<1, p \mathrm{~s}>.873$. Thus, for brevity we do not report the full analyses and focus our results on reaction times below.

Mean RT (see Table 1) was submitted to a 2 (block: baseline vs. $\mathrm{PM}) \times 2$ (word type: word vs. nonword) $\times 2$ (location: upper vs. lower) $\times 3$ (condition: specific vs. nonspecific vs. specific-70) mixed-factorial ANOVA. There was an effect of block, $F(1$, $100)=8.92, p=.004, \eta^{2}=.082$, word type, $F(1,100)=4.32$, $p=.04, \eta^{2}=.041$, and location, $F(1,100)=185.53, p<.001$, $\eta^{2}=.650$, with slower RTs for the PM block, for words, and in the upper location. The effect of condition was not significant, $F$ $<1$. However, other than the two-way interaction of word type and location, the two-way interaction of word type and condition, and the three-way interaction of block, word type, and condition, all other higher-order interactions including the four-way interaction were significant, Block $\times$ Word Type: $F(1,100)=151.84$, $p<.001, \eta^{2}=.603$; Block $\times$ Location: $F(1,100)=98.06, p<$ $.001, \eta^{2}=.495$; Block $\times$ Condition: $F(2,100)=8.24, p<.001$, $\eta^{2}=.141$; Word Type $\times$ Location: $F<1$; Word Type $\times$ Condition: $F(2,100)=1.28, p=.284, \eta^{2}=.025$; Location $\times$ Condition: $F(2,100)=23.40, p<.001, \eta^{2}=.319$; Block $\times$ Word Type $\times$ Location: $F(1,100)=31.31, p<.001, \eta^{2}=.238$; Block $\times$ Word Type $\times$ Condition: $F<1$; Block $\times$ Location $\times$ Condition: $F(2,100)=31.78, p<.001, \eta^{2}=.389$; Word Type $\times$ Location $\times$ Condition: $F(2,100)=10.07, p<.001, \eta^{2}=.168$; Block $\times$ Word Type $\times$ Location $\times$ Condition: $F(2,100)=10.47, p<.001, \eta^{2}=$ .173 . In subsequent analyses, we decompose the four-way interaction to examine whether participants were sensitive to task instructions in their monitoring of PM targets.

Of primary interest was comparing cost across expected and unexpected contexts for the various conditions (see Fig. 6). Thus, we performed separate analyses for each of the four contexts (i.e., upper word, upper nonword, lower word, lower nonword) by submitting mean RTs to a 2 (block: baseline vs. PM) $\times 3$ (condition: specific vs. nonspecific vs. specific-70) mixed-factorial ANOVA. To foreshadow, participants in the nonspecific and specific-70 conditions monitored similarly across all contexts, whereas those in the specific condition reduced monitoring in unexpected contexts.

Upper word (expected context) For word RTs in the upper location, there was an effect of block, $F(1,100)=84.60, p<$ $.001, \eta^{2}=.458$, with slower RTs in the PM block. However, there was no effect of condition and no interaction of block and condition, $F \mathrm{~s}<1$. The null interaction reflects that cost was similar between specific $(M=102 \mathrm{~ms}, S E=16 \mathrm{~ms})$, nonspecific $(M=106 \mathrm{~ms}, S E=22 \mathrm{~ms})$, and specific- ${ }_{70}(M=$ $106 \mathrm{~ms}, S E=21 \mathrm{~ms}$ ) conditions.

Lower word (unexpected context) For word RTs in the lower location, there was an effect of block, $F(1,100)=61.20, p<$ $.001, \eta^{2}=.380$, no effect of condition, $F(2,100)=2.27, p=$ 


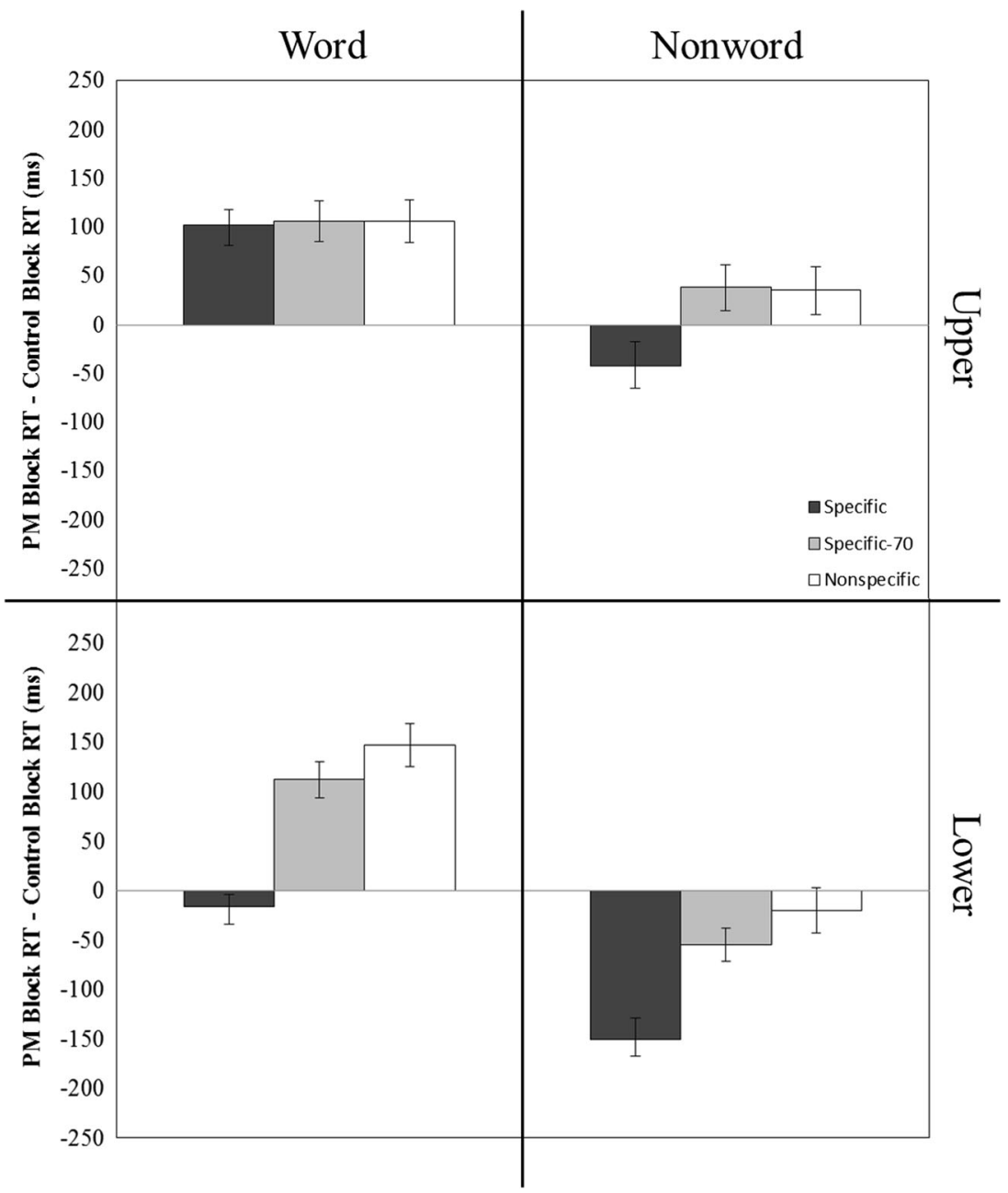

Fig. 6 Cost estimates plotted separately by word type (word, nonword) and location (upper, lower) for each condition in Experiment 3

$.109, \eta^{2 p}=.043$, and a significant interaction of block and condition, $F(2,100)=23.16, p<.001, \eta^{2}=.317$. This interaction reflects that cost was greater in the specific-70 $(M=$ $112 \mathrm{~ms}, S E=18 \mathrm{~ms})$ than the specific $(M=-16 \mathrm{~ms}, S E=$ $12 \mathrm{~ms}$ ) condition, $F(1,68)=34.43, p<.001, \eta^{2 p}=.336$, but there was no difference between the specific-70 and nonspecific $(M=147 \mathrm{~ms}, S E=22 \mathrm{~ms})$ conditions, $F(1,66)=1.46, p$ $=.231, \eta^{2 p}=.022$.

Upper nonword (unexpected context) For nonword RTs in the upper location, there was no effect of block or condition, $F \mathrm{~s}<1$. However, there was a significant interaction of block and condition, $F(2,100)=3.62, p$ $=.03, \eta^{2 p}=.068$. This interaction reflects that cost was greater in the specific- $70(M=38 \mathrm{~ms}, S E=23 \mathrm{~ms})$ than the specific $(M=-42 \mathrm{~ms}, S E=24 \mathrm{~ms})$ condition, $F(1$, $68)=5.57, p=.021, \eta^{2 p}=.076$, but there was no difference between the specific-70 and nonspecific ( $M$ $=35 \mathrm{~ms}, S E=24 \mathrm{~ms}$ ) conditions, $F<1$.
Lower nonword (unexpected context) For nonword RTs in the lower location, there was an effect of block, $F(1,100)=$ $39.21, p<.001, \eta^{2}=.282$, no effect of condition, $F(2,100)=$ $1.89, p=.156, \eta^{2}=.036$, and a significant interaction of block and condition, $F(2,100)=10.65, p<.001, \eta^{2 p}=.176$. This interaction reflects that cost was greater in the specific-70 $(M=-55 \mathrm{~ms}, S E=17 \mathrm{~ms})$ than the specific $(M=-151 \mathrm{~ms}, S E=$ $22 \mathrm{~ms}$ ) condition, $F(1,68)=12.10, p=.001, \eta^{2 p}=.151$, but there was no difference between the specific-70 and nonspecific $(M=-20 \mathrm{~ms}, S E=23 \mathrm{~ms})$ conditions, $F(1,66)=1.46, p=$ $.232, \eta^{2}=.022$.

PM target detection in expected context To examine PM performance, the proportion of successfully detected PM targets in the upper word context was submitted to a between-subjects ANOVA (right hand portion of Fig. 3). This analysis revealed a significant effect of condition, $F(2,100)=3.60, p=.031, \eta^{2 p}=$ .067 , indicating reduced performance for the nonspecific $(M=$ $.75, S E=.06)$ relative to the specific-70 $(M=.90, S E=.02)$ 
condition, $F(1,66)=5.73, p=.02, \eta^{2 p}=.08$. There was no difference between the specific condition $(M=.86, S E=.03)$ and either of the other two conditions, $F \mathrm{~s}<2.58, p>.114$.

Notably, the reduced performance in the nonspecific condition was driven by four participants that did not detect any PM targets. If these participants are excluded from the analyses, there are no differences across conditions, $F<1$, and target detection in the nonspecific condition $(M=.85, S E=$ $.04)$ is comparable to the other two conditions. ${ }^{4}$ It should be noted, however, that these participants did correctly recall the PM instructions ("press '7' upon encountering 'tor' syllable") during a post-experimental questionnaire and prior research typically only excludes participants if they fail to detect any targets and are unable to recall the PM instructions on a postexperimental questionnaire (as this reflects a retrospective rather than a prospective memory failure; Zimmermann \& Meier, 2006). However, as described in the Method section in the current experiment, prior to administration of the postexperimental questionnaire we asked participants if they had seen "tor" in the last five trials of the task and if they responded with the "7" key upon encountering it, which may have influenced answers on the postexperimental questionnaire. Nevertheless, given that the two previous experiments, other unpublished research from our laboratory, and prior research (Lourenço et al., 2014) has failed to find target detection differences following context instructions, we do not wish to make strong claims about the difference between nonspecific and specific-70 conditions (which were not significantly different in Experiment 2).

PM target detection in unexpected context To examine PM target detection in the unexpected context, the number of participants that responded to the PM target in the final (lower nonword) block was submitted to a chi-square test (right hand portion of Fig. 4). This analysis revealed a significant difference in the number of participants that detected targets across conditions, $\chi^{2}(1, N=103)=44.49, p<.001$. Fewer participants in the specific condition $(0 \%)$ detected the final target than the specific- ${ }_{70}$ condition $(60 \%), \chi^{2}(1, N=70)=30.0, p<$ .001 , but there was no difference between the specific-70 and nonspecific (76\%) conditions, $\chi^{2}(1, N=68)=1.93, p=.165$.

We also examined whether monitoring cost (PM block control block RTs) in the lower nonword context was predictive of target detection (collapsed across conditions). As expected, a logistic regression revealed that individuals that exhibited greater cost were more likely to detect the final target in the unexpected context, Wald $\chi^{2}(1, N=103)=18.09, p<$ .001 .

In addition to examining the proportion of participants correctly responding to the final target in the unexpected context,

\footnotetext{
${ }^{4}$ Note that the exclusion of these participants does not significantly change any of the reported RT results.
}

the current study also examined the alternative hypothesis that participants in the specific condition indeed noticed the target but simply did not respond because they thought the instructions implied that they should respond to targets in the upper word context only. Thus, as described previously, immediately following the final lexical decision participants were asked whether or not they saw an item with the "tor" syllable in the last five trials of the task. If so, participants were to indicate whether or not they responded with the "7" key, and participants that indicated that they did not respond were subsequently asked to report why they withheld the response. As can be seen in the top row of Table 2, only 11 of the 35 participants in the specific condition reported having seen the final target. In contrast, participants in the specific-70 $(25$ of 35) and nonspecific (22 of 33) conditions more often claimed to see the target. A chi-square test confirmed there was a significant difference in the number of participants claiming to notice the target across conditions, $\chi^{2}(1, N=$ $103)=13.50, p=.001$. Fewer participants in the specific condition $(31 \%)$ claimed to notice the final target than the nonspecific condition $(67 \%), \chi^{2}(1, N=68)=8.44, p=.004$, but there was no difference between the nonspecific and specific- ${ }_{70}$ conditions $(71 \%), \chi^{2}(1, N=68)=.18, p=.671$.

Table 2 further breaks down participant reports conditional on whether or not they actually responded to the final target during the ongoing task separately for each condition. The findings from the specific- 70 and nonspecific conditions suggest that participants were generally accurate in their self-reports, such that those that responded during the ongoing task generally remembered doing so when questioned (and vice versa), although this was not perfect. However, of primary importance for the current study was to examine why participants in the specific condition did not respond upon noticing the target. Interestingly, of the 11 participants claiming to see the final target, five of these participants incorrectly indicated having actually responded to the final target during the ongoing task. Of the remaining six participants that noticed the target and correctly indicated that they did not respond to it, four reported that the reason they did not respond was because it was in a nonword or in the lower location (or both), whereas the other two participants indicated that they were going too quickly on the ongoing task and missed it. These findings suggest that there were indeed a few (four) participants in the specific condition that thought the instructions implied that they should not respond to targets in the lower or nonword contexts, but this made up only a small percentage of individuals.

\section{Discussion}

There were two primary findings in Experiment 3. First, using a design that directly contrasted PM performance in response 
Table 2 Summary of responses to the questions that queried participants about their noticing of and response to PM target presented in the unexpected context (final lower nonword block) in Experiment 3

\begin{tabular}{|c|c|c|c|c|c|c|}
\hline & \multicolumn{6}{|l|}{ Condition } \\
\hline & $\begin{array}{l}\text { Specific } \\
\text { Count }\end{array}$ & $\%$ & $\begin{array}{l}\text { Specific-70 } \\
\text { Count }\end{array}$ & $\%$ & $\begin{array}{l}\text { Nonspecific } \\
\text { Count }\end{array}$ & $\%$ \\
\hline \multicolumn{7}{|l|}{ Overall } \\
\hline Claimed to see target & 11 of 35 & 0.31 & 25 of 35 & 0.67 & 22 of 33 & \\
\hline Did not respond & 35 of 35 & 1.00 & 14 of 35 & 0.40 & 8 of 33 & 0.24 \\
\hline Claimed to see target & 11 of 35 & 0.31 & 7 of 14 & 0.50 & 4 of 8 & 0.50 \\
\hline Correctly claimed to not respond & 6 of 11 & 0.55 & 5 of 7 & 0.70 & 1 of 4 & 0.25 \\
\hline Did Respond & - & - & 21 of 35 & 0.60 & 25 of 33 & 0.76 \\
\hline Claimed to see target & - & - & 18 of 21 & 0.86 & 18 of 25 & 0.72 \\
\hline Correctly claimed to respond & - & - & 18 of 18 & 1.00 & 16 of 18 & 0.89 \\
\hline
\end{tabular}

to a deterministic (specific condition) and probabilistic (specific $_{-70}$ condition) complex contextual cue, it was demonstrated that strategic monitoring was evidenced selectively for the deterministic contextual cue. In the expected context, all three conditions (specific, specific-70, and nonspecific) monitored equivalently, as anticipated. However, in all three of the unexpected contexts, the specific condition relaxed monitoring relative to the nonspecific condition whereas the specific $_{-70}$ condition did not. The specific $_{-70}$ condition monitored as much as the nonspecific condition and significantly more than the specific condition in each of the unexpected contexts. These findings provide strong support for the conclusion that the predictive validity of the complex contextual cue limits use of strategic monitoring, thereby representing a potential boundary condition. When it was $70 \%$ likely that the PM target would occur in a particular context, participants did not engage monitoring selectively in that context. Only when it was $100 \%$ likely was strategic monitoring observed in response to the complex contextual cue.

The second primary finding stemmed from our inquiry into the origin of the very low rate of PM target detection in the unexpected context for the specific condition in Experiment 1. The question was whether this represented a true disadvantage of strategic monitoring in the specific condition (i.e., a detriment to performance associated with the relaxation of monitoring) or participants' belief that they should not respond to PM targets outside of the expected context. Replicating Experiment 1, significantly fewer participants responded to the PM target in the specific compared to the nonspecific condition. With respect to the specific $\mathrm{c}_{-70}$ condition, considering that this condition did not show evidence of strategic monitoring and cost patterns resembled the nonspecific condition, the prediction was that PM target detection in the unexpected context should be higher for the specific s $_{-70}$ condition than the specific condition but should be equivalent to the nonspecific condition. This prediction was confirmed.
Turning to the novel data from the post-target query, critically, significantly fewer participants in the specific condition reported noticing the PM target in the unexpected context relative to either the nonspecific or specific -70 condition (which did not differ). Furthermore, of the 35 participants in the specific condition, only four of them claimed to have noticed but withheld a response to the PM target because they thought they should not respond to targets that were nonwords or in a lower location. These patterns collectively support the conclusion that there is a disadvantage associated with strategic monitoring (i.e., for the specific condition), and this disadvantage is attributable to the reduced tendency to notice PM targets in unexpected contexts in which monitoring has been relaxed.

\section{General discussion}

This study investigated potential boundary conditions for strategic monitoring. Experiment 1 provided novel evidence for strategic monitoring in response to a complex contextual cue. A specific condition that was led to expect PM targets solely in the upper word context monitored equivalently to a nonspecific condition that was not provided information about the contextual cue, selectively within that context. In the unexpected contexts, the specific condition relaxed monitoring relative to the nonspecific condition, and monitoring was absent for the specific condition in the lower nonword context that shared no overlapping features with the contextual cue. These patterns demonstrate that strategic monitoring is not necessarily limited to provision of simple contextual cues that are defined by a single feature (e.g., color, Lourenço \& Maylor, 2014; word type, Lourenço et al., 2013; shape, Kuhlmann \& Rummel, 2014) but may also be observed in response to complex contextual cues. Use of complex contextual cues per se is thus not a boundary condition for strategic monitoring. 
The findings of Experiment 2, in contrast, did suggest a potential boundary condition for strategic monitoring, namely use of probabilistic contextual cues. Using the same blocked PM procedure as in Experiment 1 that placed minimal demands on attention in the service of detecting the appropriate contexts to heighten or relax monitoring, strategic monitoring was largely not evidenced in the probabilistic complex cue conditions ( specific $_{-90}$ and specific $_{-70}$ ). Experiment 3 provided stronger evidence that the predictive validity of the contextual cue affects strategic monitoring by directly contrasting a deterministic (specific) and probabilistic ( specific $_{-70}$ ) complex cue condition. Strategic monitoring was evidenced selectively for the deterministic cue. While these findings call into question the ubiquity of strategic monitoring as a means of heightening processes such as target checking (when needed) and conserving resources (when possible) in response to probabilistic contextual cues, they do fit with models positing that decisions to utilize costly cognitive resources (such as those that appear to support strategic monitoring; see, e.g., Lourenço $\&$ Maylor, 2014) factor in the required effort and expected reward (Shenhav et al., 2013). The reluctance to engage strategic monitoring processes to detect and modulate the extent to which a retrieval mode is initiated or target checking is engaged may reflect that the cost/benefit ratio did not justify the effort required to engage these resources (e.g., Shenhav et al., 2013). That is, while there was an expected benefit to PM target detection to justify monitoring in the upper word context for the specific -70 condition (albeit not as great as in the specific condition), the possibility of missing a PM target in the unexpected contexts was perhaps too high to justify a relaxation of monitoring (or to switch between a heightened and relaxed mode of monitoring).

It is interesting to consider the relevance of the current findings to extant theoretical accounts of strategic monitoring. The findings from the specific condition in Experiments 1 and 3 align with the "attentional control" account of strategic monitoring (Lourenço \& Maylor, 2014; Lourenço et al., 2013), which proposes that the PM demands set forth by the instructions in the specific condition lead participants to allocate more attention to target checking when in an expected as compared to unexpected context, thereby freeing up resources for engaging in the ongoing task in the unexpected context (see also Guynn, 2003). This account posits that strategic monitoring depends on limited-capacity attentional resources, namely in service of identifying a particular context on a given trial and adjusting how much attention is allocated to monitoring for the PM target from one trial to the next.

The delay theory of PM (Heathcote, Loft, \& Remington, 2015; Loft \& Remington, 2013) recently provided an alternative to the attentional control account, and it too may accommodate the findings of Experiments 1 and 3 . According to this theory, costs arise because the PM task races and competes for response selection with the more routine ongoing task, and to ensure that an ongoing task response is not made in lieu of a PM response, participants selectively delay responding to allow more time for PM evidence to accumulate. Consistent with this idea, Heathcote et al. modeled the data of Lourenço et al. (2013) and found evidence that participants selectively delayed responding within the expected context (i.e., on word trials) in the specific condition. This theory contrasts with the attentional control view of Lourenço et al. in that the delay theory suggests that participants simply set a bias to delay responding after learning the relationship between a particular context and the occurrence of PM targets and therefore do not need to engage attentionally demanding, strategic target checks on a trial-bytrial basis to support prospective remembering.

The findings of Experiment 2 and Experiment 3, which point to the use of costly attentional resources in guiding strategic monitoring, may also be accommodated by the attentional control view of Lourenço and colleagues (Lourenço \& Maylor, 2014; Lourenço et al., 2013), as participants appeared to be reluctant to deploy these resources with use of the probabilistic contextual cues. Applying delay theory (Heathcote et al., 2015; Loft \& Remington, 2013), it could be suggested that participants were disinclined to adjust their response bias in response to the probabilistic contextual cues; however, it is uncertain if establishing or adjusting a response bias demands costly attentional resources.

A final finding that was observed in the current experiments pertained to our examination of a potential disadvantage of relaxing monitoring processes in unexpected contexts. In a final block, a PM target was embedded within an unexpected (lower nonword) context that shared no overlap with the expected, upper word context. In Experiment 1 where evidence for the relaxation of monitoring was robust in the specific condition, only 5\% of participants detected the PM target compared to $76 \%$ of participants in the nonspecific condition. This difference supported the view that the relaxation of monitoring may come at the expense of missing unexpected but imperative stimuli, a pattern that converges with the results from a time-based PM procedure of Cook et al. (2005). In Experiment 2 where evidence for relaxation of monitoring was weak (specific-90) or absent (specific-70), the PM target detection data were less clear. Target detection was lower for the specific-90 condition relative to the nonspecific condition. However, the specific-70 condition fell in the middle and did not differ from the other conditions. In both experiments, collapsing across conditions, the degree of monitoring (as indicated by the magnitude of cost) in the unexpected, lower nonword context was positively associated with PM target detection in that context.

Although the above findings are fairly consistent with the view that the relaxation of monitoring in unexpected contexts (i.e., strategic monitoring, as in the specific condition) comes 
at a disadvantage to performance (reduced PM target detection in unexpected contexts), Experiments 1 and 2 could not rule out an alternative account. According to the alternative account, the disadvantage of relaxing monitoring in the specific condition may have been overestimated if participants interpreted the instructions to mean they should respond to PM targets in the expected (upper word) context only. Experiment 3 tested this account by querying participants about their noticing of the final PM target and their decision to respond (or not to respond) shortly after the target was presented in the unexpected (lower nonword) context. Countering the alternative account, participants in the specific condition were not only less likely to respond to the PM target in the unexpected context but they were also less likely to report having noticed the PM target (relative to the nonspecific and specific $_{-70}$ conditions). Finally, only a few participants in the specific condition reported that they noticed but did not respond to the PM target in the unexpected context specifically because they thought they should not respond to it. These data converge on the conclusion that although strategic monitoring is advantageous (i.e., permits conservation of limited attentional resources), there is also a disadvantage associated with strategic monitoring (i.e., reduced sensitivity to PM targets outside of the expected context).

\section{Limitations and future directions}

One potential limitation that should be noted concerns the blocked PM procedure employed in the present study. Because contextual cues were blocked by word type (in addition to location) and the ongoing task was lexical decision, participants could theoretically register a lexical decision response without fully processing stimuli (to determine lexicality) once the context was identified. The fact that context changes were not signaled to participants may have deterred this strategy. However, even if it was employed, several observations counter the view that the present findings (e.g., strategic monitoring in response to a complex contextual cue) are an artifact of this design feature. First, the overall pattern of strategic monitoring observed in Experiment 1 (and for the specific and nonspecific conditions in Experiment 3) coincides with that observed in the original variant of the blocked PM procedure that did not include this design feature but also used a deterministic contextual cue (e.g., Lourenço \& Maylor, 2014). Second, this feature was present for all participants, not just those in the specific condition, yet there were still differing patterns of monitoring across conditions. Third, monitoring was never eliminated in the specific condition (with the exception of one context, though this finding was not anticipated by prior findings with the blocked PM procedure or theory, and was not replicated in a separate study), which suggests participants were still engaging monitoring processes. Finally, the strategic monitoring pattern was observed selectively in the specific condition and not the specific s $_{90}$ or specific -70 conditions, which used the same blocking procedure.

The current findings point to several fruitful avenues for future research. First, the findings of Experiment 1 suggest use of complex contextual cues is not necessarily a boundary condition for strategic monitoring. However, this conclusion may depend on use of the blocked PM procedure. Strategic monitoring may not be observed with use of the random PM procedure that requires participants to allocate attention to identifying whether a context is expected versus unexpected on a trial-by-trial basis. The conclusion may also depend on the particular type of complex contextual cue used in the current study. Research on basic properties of attention has highlighted that some features such as location can be detected quickly and relatively automatically upon stimulus onset (Logan, 1998; Mayr, 1996; cf. Crump, Gong, \& Milliken, 2006), whereas detection of others such as stimulus identity places demands on attention poststimulus onset (Besner, Risko, \& Sklair, 2005; McCann, Folk, \& Johnston, 1992). Strategic monitoring may not be observed in the blocked PM procedure if the two contextual dimensions both require attentional resources post-stimulus onset for context identification (e.g., word type and shape). Investigation of these issues will continue to help clarify the importance of attentional resources in guiding strategic monitoring (cf. Lourenço \& Maylor, 2014).

Second, although Experiments 2 and 3 demonstrate that use of probabilistic contextual cues may represent a boundary condition for strategic monitoring, it remains possible that this conclusion is limited to complex contextual cues that are probabilistic. Individuals may justify the effort to monitor strategically in spite of there being less clear benefits when the attentional demands of doing so are fairly minimal, as may be the case with simple contextual cues. Finally, future studies might examine whether instructions or incentives that vary the importance of detecting targets in expected contexts versus missing PM targets in unexpected contexts influence patterns of strategic monitoring in response to probabilistic contextual cues (cf. Bugg, Diede, Cohen-Shikora, \& Selmeczy, 2015).

\section{Conclusion}

The results from the current study suggest that participants use complex contextual cues to guide strategic monitoring, at least when strong environmental support is available to facilitate context identification (i.e., blocked PM procedure). Strategic monitoring reduces unnecessary allocation of attention in contexts in which PM targets are not expected to occur. However, strategic monitoring is not evidenced when a complex contextual cue is probabilistic. These results highlight the importance 
of costly, limited-capacity attentional resources in guiding strategic monitoring. The results may also be of practical significance, as intentions established in everyday life may be complex (i.e., comprise multiple contextual dimensions), and the occurrence of PM targets in particular contexts may often be probabilistic. Consequently, to ensure that PM intentions (e.g., remembering to deliver a message to a professor) are successfully fulfilled, it may be advisable to engage monitoring processes to some degree even in contexts in which encountering PM targets may be less likely. Indeed, this would minimize the potential disadvantage (i.e., failing to notice the professor in the unexpected context, resulting in failure to deliver the message) of strategic monitoring that was evidenced in our study.

Author note Julie M. Bugg and B. Hunter Ball, Department of Psychological and Brain Sciences, Washington University in St. Louis, CB1125, One Brookings Dr., St. Louis, MO, 63130.

The authors are grateful to Suzanna Powell who assisted with programming and data collection, and Raveena Boodram, Jaelyn DeBerry, Whitney Dominguez, Guhan Iyer, Peeta Li, Lela Pritchett, and Jenny Weissman, who assisted with data collection. B.H.B. was supported by an NIA Training Grant (T32AG000030-40). Portions of the data were presented at the 87th Annual Meeting of the Midwestern Psychological Association, Chicago, IL.

\section{References}

Ball, B. H., Brewer, G. A., Loft, S., \& Bowden, V. (2014). Uncovering continuous and transient monitoring profiles in event-based prospective memory. Psychonomic Bulletin \& Review, 22(2), 492-499.

Balota, D.A., Yap, M.J., Cortese, M.J., Hutchison, K.A., Kessler, B., Loftis, B.,...Treiman, R. (2007). The english lexicon project. Behavior Research Methods, 39, 445-459.

Besner, D., Risko, E. F., \& Sklair, N. (2005). Spatial attention as a necessary preliminary to early processes in reading. Canadian Journal of Experimental Psychology/Revue canadienne de psychologie expérimentale, 59(2), 99.

Bugg, J. M., Diede, N. T., Cohen-Shikora, E., \& Selmeczy, D. (2015). Expectations and experience: Dissociable bases for cognitive control? Journal of Experimental Psychology: Learning, Memory, and Cognition, 41, 13491373.

Bugg, J. M., \& Smallwood, A. (2016). The next trial will be conflicting! The effects of explicit congruency pre-cues on cognitive control. Psychological Research, 80, 16-33.

Cohen, A. L., Jaudas, A., Hirschhorn, E., Sobin, E., \& Gollwitzer, P. M. (2012). The specificity of prospective memory costs. Memory, 20, 848-864.

Cook, G. I., Marsh, R. L., \& Hicks, J. L. (2005). Associating a time-based prospective memory task with an expected context can improve or impair intention completion. Applied Cognitive Psychology, 19, 345-360.

Crump, M. J., Gong, Z., \& Milliken, B. (2006). The context-specific proportion congruent Stroop effect: Location as a contextual cue. Psychonomic Bulletin \& Review, 13(2), 316-321.

Einstein, G. O., \& McDaniel, M. A. (2005). Prospective memory: Multiple retrieval processes. Current Directions in Psychological Science, 14, 286-290.

Einstein, G. O., McDaniel, M. A., Williford, C. L., Pagan, J. L., \& Dismukes, R. K. (2003). Forgetting of intentions in demanding situations is rapid. Journal of Experimental Psychology: Applied, 9. $147-162$.

Guynn, M. J. (2003). A two-process model of strategic monitoring in event-based prospective memory: Activation/retrieval mode and checking. International Journal of Psychology, 38, 245-256.

Heathcote, A., Loft, S., \& Remington, R. W. (2015). Slow down and remember to remember! A delay theory of prospective memory costs. Psychological Review, 122(2), 376.

Knight, J. B., Meeks, J. T., Marsh, R. L., Cook, G. I., Brewer, G. A., \& Hicks, J. L. (2011). An observation on the spontaneous noticing of prospective memory event-based cues. Journal of Experimental Psychology: Learning, Memory, and Cognition, 37, 298-307.

Kuhlmann, B. G., \& Rummel, J. (2014). Context-specific prospectivememory processing: Evidence for flexible attention allocation adjustments after intention encoding. Memory \& Cognition, 42(6), 943-949.

Loft, S., \& Remington, R. W. (2013). Brief delays in responding reduce focality effects in event-based prospective memory. The Quarterly Journal of Experimental Psychology, 66, 1432-1447.

Logan, G. D. (1998). What is learned during automatization? II. Obligatory encoding of spatial location. Journal of Experimental Psychology: Human Perception and Performance, 24(6), 1720.

Lourenço, J. S., \& Maylor, E. A. (2014). Is it relevant? Influence of trial manipulations of prospective memory context on task interference. The Quarterly Journal of Experimental Psychology, 67(4), 687702.

Lourenço, J. S., White, K., \& Maylor, E. A. (2013). Target context specification can reduce costs in nonfocal prospective memory. Journal of Experimental Psychology: Learning, Memory, and Cognition, 39(6), 1757.

Marsh, R. L., Cook, G. I., \& Hicks, J. L. (2006). Task interference from event-based intentions can be material specific. Memory \& Cognition, 34, 1636-1643.

Marsh, R. L., Hicks, J. L., \& Cook, G. I. (2006). Task interference from prospective memories covaries with contextual associations of fulfilling them. Memory \& Cognition, 34(5), 1037-1045.

Mayr, U. (1996). Spatial attention and implicit sequence learning: Evidence for independent learning of spatial and nonspatial sequences. Journal of Experimental Psychology: Learning, Memory, and Cognition, 22(2), 350.

McCann, R. S., Folk, C. L., \& Johnston, J. C. (1992). The role of spatial attention in visual word processing. Journal of Experimental Psychology: Human Perception and Performance, 18(4), 10151029.

McDaniel, M. A., \& Einstein, G. O. (2000). Strategic and automatic processes in prospective memory retrieval. Applied Cognitive Psychology, 14, S127-S144.

Meier, B., Zimmermann, T. D., \& Perrig, W. J. (2006). Retrieval experience in prospective memory: Strategic monitoring and spontaneous retrieval. Memory, 14(7), 872-889.

Prinzmetal, W., Presti, D. E., \& Posner, M. I. (1986). Does attention affect feature integration? Journal of Experimental Psychology: Human Perception and Performance, 12, 361-369.

Scullin, M. K., McDaniel, M. A., \& Shelton, J. T. (2013). The dynamic multiprocess framework: Evidence from prospective memory with contextual variability. Cognitive Psychology, 67(1), 55-71.

Shenhav, A., Botvinick, M. M., \& Cohen, J. D. (2013). The expected value of control: An integrative theory of anterior cingulate cortex function. Neuron, 79(2), 217-240.

Shipley, W. C. (1940). A self-administering scale for measuring intellectual impairment and deterioration. The Journal of Psychology, 9, 371-377.

Smith, R. E. (2003). The cost of remembering to remember in eventbased prospective memory: Investigating the capacity demands of 
delayed intention performance. Journal of Experimental Psychology: Learning, Memory, \& Cognition, 29, 347-361.

Smith, R. E. (2016). Prospective Memory in Context. Psychology of Learning and Motivation, 66, 211-249.

Smith, R. E., \& Bayen, U. J. (2004). A multinomial model of event-based prospective memory. Journal of Experimental Psychology: Learning, Memory, and Cognition, 30(4), 756.

Smith, R. E., Hunt, R. R., \& Murray, A. E. (2016). Prospective memory in context: Moving through a familiar space. Journal of Experimental
Psychology: Learning, Memory, \& Cognition. Advance online publication. doi:10.1037/xlm0000303

Treisman, A. M., \& Gelade, G. (1980). A feature-integration theory of attention. Cognitive Psychology, 12(1), 97-136.

Zimmermann, T. D., \& Meier, B. (2006). The rise and decline of prospective memory performance across the lifespan. The Quarterly Journal of Experimental Psychology, 59(12), 2040-2046. 